\title{
Unbiased integration of single cell multi-omics data
}

Jinzhuang Dou¹,jidou1@mdanderson.org

Shaoheng Liang1, sliang3@mdanderson.org

Vakul Mohanty릴. vmohanty@mdanderson.org

Xuesen Cheng², xuesenc@bcm.edu

Sangbae Kim², $\underline{\text { Sangbae.Kim } @ \text { bcm.edu }}$

Jongsu Choi², Jongsu.Choi@bcm.edu

Yumei Li², yumeil@bcm.edu

Katayoun Rezvani ${ }^{4}$, krezvani@mdanderson.org

Rui Chen ${ }^{2,3}$, ruichen@bcm.edu

Ken Chen ${ }^{1, *}$. kchen3@mdanderson.org

${ }^{1}$ Department of Bioinformatics and Computational Biology, The University of Texas MD Anderson Cancer Center

${ }^{2}$ HGSC, Department of Molecular and Human Genetics, Baylor College of Medicine, Houston, TX, 77030, USA

${ }^{3}$ Verna and Marrs McLean Department of Biochemistry and Molecular Biology, Baylor College of Medicine,

Houston, TX, 77030, USA

${ }^{4}$ Department of Stem Cell Transplantation and Cellular Therapy, The University of Texas MD Anderson Cancer

Center, Houston, Texas

* Correspondence: kchen3@mdanderson.org 


\section{Abstract}

2 Acquiring accurate single-cell multiomics profiles often requires performing unbiased in silico

3 integration of data matrices generated by different single-cell technologies from the same

4 biological sample. However, both the rows and the columns can represent different entities in

5 different data matrices, making such integration a computational challenge that has only been

6 solved approximately by existing approaches. Here, we present bindSC, a single-cell data

7 integration tool that realizes simultaneous alignment of the rows and the columns between data

8 matrices without making approximations. Using datasets produced by multiomics technologies as

9 gold standard, we show that bindSC generates accurate multimodal co-embeddings that are

10 substantially more accurate than those generated by existing approaches. Particularly, bindSC

11 effectively integrated single cell RNA sequencing (scRNA-seq) and single cell chromatin

12 accessibility sequencing (scATAC-seq) data towards discovering key regulatory elements in

13 cancer cell-lines and mouse cells. It achieved accurate integration of both common and rare cell

14 types $(<0.25 \%$ abundance $)$ in a novel mouse retina cell atlas generated using the $10 \mathrm{x}$ Genomics

15 Multiome ATAC+RNA kit. Further, it achieves unbiased integration of scRNA-seq and 10x

16 Visium spatial transcriptomics data derived from mouse brain cortex samples. Lastly, it

17 demonstrated efficacy in delineating immune cell types via integrating single-cell RNA and

18 protein data. Thus, bindSC, available at https://github.com/KChen-lab/bindSC, can be applied in

19 a broad variety of context to accelerate discovery of complex cellular and biological identities and

20 associated molecular underpinnings in diseases and developing organisms. 


\section{Introductions}

22 Advances in high-throughput single-cell technology such as single-cell RNA-sequencing (scRNA-

23 seq) ${ }^{1}$ and mass cytometry ${ }^{2}$ have enabled systematic delineation of cell types based on thousands

24 to millions of cells sampled from developing organisms or patient biopsies. For example, recent

25 application of combinatorial indexing based technology has generated the transcriptomic and

26 chromatin accessibility profiles of millions of cells in developing human fetus samples ${ }^{3}$. Rare cell

27 types and complex cellular states, however, remain challenging to discover, which necessitates the

28 development of multiomics technologies to simultaneously measure other cellular features,

29 including DNA methylation ${ }^{4,5}$, chromatin accessibility ${ }^{6-8}$ and spatial positions ${ }^{9,10}$ in the same

30 cells. Although available single-cell multiomics technologies ${ }^{8,11-14}$ can profile thousands to

31 millions of cells per experiment, the cost of the experiments is still quite high ${ }^{15}$; and the data

32 generated are often of lower throughput than those generated by unimodal technologies. These

33 restrictions necessitate the development of computational approaches that can accurately integrate

34 multiple data matrices generated by different technologies from the same biological samples to

35 acquire an accurate characterization of cellular identity and function.

37 However, different technologies create data matrices of different rows and columns, which

38 correspond to different sets of cells and different types of features. How to align cells and features

39 simultaneously across matrices is a core computational challenge. When the two sets of cells are

40 sampled uniformly from the same biological sample, it is safe to assume that there exists an optimal

41 way to align together cells of similar identities and features associated with these identities. This

42 is mathematically challenging, however, as there are many possible ways to simultaneously align

43 a large number of cells and features. To address this challenge, existing computational approaches 
44 followed two directions: 1) aligning features empirically before aligning cells ${ }^{16-19}$; 2) obtaining 45 separate embeddings for each modality, followed by performing unsupervised manifold alignment

$46^{20-22}$. Taking integration of scRNA-seq and singe cell assay for transposase accessible chromatin

47 sequencing (scATAC-seq) as an example, the first category of methods require constructing a

48 "gene activity matrix" from scATAC-seq data by counting DNA reads aligned near and within

49 each gene ${ }^{23}$. This strategy considers only the basic cis-regulatory relations and ignores long-range,

50 trans-regulatory relationship established via other regulatory elements such as enhancers ${ }^{6}$, which

51 are often critical to decipher cell identities. It also substantially simplifies (or loses) multifactorial

52 relations between transcription factors (TF) and target genes ${ }^{24}$. Based on pre-aligned features

53 generated by such empirical rules, Seurat applies canonical correlation analysis (CCA) and mutual

54 nearest neighbors (MNNs) to identify cells anchoring the two data matrices ${ }^{17}$; LIGER uses an

55 integrative non-negative matrix factorization (iNMF) to delineate shared and dataset-specific

56 features ${ }^{19}$; Harmony projects cells onto a shared embedding using principle components analysis

57 (PCA) and removes batch effects iteratively ${ }^{18}$. All these programs suffer from the aforementioned

58 limitations and thereby cannot yield a comprehensive, unbiased gene regulatory network,

59 particularly when chromatin changes are asynchronous from RNA transcriptions in cells

60 undergoing state transitions ${ }^{25}$. The second category of methods ${ }^{20-22}$ do not require prior feature

61 alignment and are fully unsupervised. However, they depend heavily on the assumption that

62 feature variation across cells is driven by a few latent variables in both modalities ${ }^{22}$. This

63 assumption can get violated easily in datasets of complex biology involving dynamic processes

64 such as differentiation, reprogramming and transdifferentiation ${ }^{22}$. 
66 In this study, we develop a novel computational tool called bindSC (bi-order integration of single-

67 cell data). The key algorithm implemented in bindSC is called bi-CCA (bi-order canonical

68 correlation analysis). Bi-CCA learns the optimal alignment among rows and columns from two

69 data matrices generated by two different experiments. The alignment matrix derived from bi-CCA

70 can thereby be utilized to derive in silico multiomics profiles from aligned cells.

72 We assess our method on several challenging multimodality integration tasks between 1)

73 transcriptomic and chromatin accessibility data, 2) transcriptomic and spatial transcriptomic data,

74 and 3) transcriptomic and proteomic data. We validate scRNA-seq and scATAC-seq integration

75 accuracy using datasets obtained directly from multiomics technologies, including a novel mouse

76 retina cell atlas created by the 10x Genomics Multiome ATAC+RNA kit. We show that bindSC

77 enables comprehensive characterization of epigenetic regulatory states in a lung adenocarcinoma

78 cell-line A549 in response to dexamethasone treatment. And bindSC can align mouse retina cell

79 types accurately, for multi-subtype bipolar cells and rare horizontal cells. Moreover, bindSC

80 enables unbiased integration of spatial transcriptomics data with scRNA-seq data on mouse brain

81 cortex samples, as well as single-cell RNA data with protein data from peripheral blood

82 mononuclear cells. BindSC is implemented as an open-source $\mathrm{R}$ package available at

83 https://github.com/KChen-lab/bindSC.

\section{Results}

87 Bi-order integration of multi-omics data 
BindSC takes as input two single-cell data matrices $(\mathbf{X}$ and $\mathbf{Y})$ generated uniformly from the same cell population by two different technologies (Fig. 1a). In most single-cell multi-omics integration tasks, neither the alignment between the cells in $\mathbf{X}$ and those in $\mathbf{Y}$, nor the alignment between the

91 features in $\mathbf{X}$ and those in $\mathbf{Y}$ is known. BindSC employs a bi-CCA algorithm developed in this

92 study to address this challenge (Fig. 1b). Briefly, bi-CCA introduces a gene score matrix $\mathbf{Z}$ to link

$93 \mathbf{X}$ and $\mathbf{Y}$. The gene score matrix has the same rows as does $\mathbf{X}$ and the same columns as does $\mathbf{Y}$. To

94 reduce computational cost, $\mathbf{Z}$ can be initialized based on prior knowledge. Taking integration of

95 scRNA-seq and scATAC-seq as an example, the gene score matrix can be initialized using the

96 "gene activity matrix" estimated by other programs such as Seurat. Bi-CCA then iteratively

97 updates $\mathbf{Z}$ to find an optimal solution which maximizes the correlation between $\mathbf{X}$ and $\mathbf{Z}$ and

98 between $\mathbf{Y}$ and $\mathbf{Z}$ in the latent space simultaneously. Details about this iterative procedure can be found in Methods and Supplementary Fig. 1a.

Bi-CCA outputs canonical correlation vectors (CCVs), which project cells from two datasets onto a shared latent space (referring below as "co-embedding"). A K-nearest neighbor (KNN) graph is constructed based on Euclidean distances observed in the latent space, followed by modularity optimization techniques to partition the KNN into highly interconnected subgraphs, each of which corresponds to a putative cell type or state (Fig. 1c). Within each cluster, sub-clustering using

106 similar strategies is further performed to derive what we call pseudo-cells (Methods). Each

107 pseudo-cell encloses tens of cells from both datasets and thus has a consensus multiomic profile 108 summarized from constituting cells (Fig. 1c-d). The joint multiomic profiles thus enable 1) 109 characterizing gene and chromatin-accessibility relations from aligned scRNA-seq and scATAC110 seq data; 2) associating transcriptomic profiles with spatial locations from aligned scRNA-seq and 
111 spatial transcriptomic data; 3) associating transcriptomic profiles with proteomic profiles from

112 aligned scRNA-seq and CyTOF data, and so on (Fig. 1e).

\section{Benchmarking bindSC performance on simulation datasets}

115 Existing integration methods such as Seurat, LIGER, and Harmony require pre-aligning features

116 across modalities, i.e., compressing cell-peak matrices obtained from scATAC-seq onto cell-gene-

117 activity matrices based on reference genome annotations. BindSC overcomes that restriction: its

118 generic mathematical formulations allow free alignment amongst features to be established from 119 data.

121 Under our formulation (Methods), $\mathbf{Z}$ has features (rows) aligned with $\mathbf{X}$ and cells (columns)

122 aligned with $\mathbf{Y}$. The introduction of $\mathbf{Z}$ enables bi-order alignment of the cells and the features, 123 respectively.

125 To quantify how much this step matters to overall integration accuracy, we performed a set of 126 simulation experiments. We started by creating a dataset $\mathbf{X}$ consisting of 3 cell clusters (types), 127 each having 333 cells and 1,000 genes using Splatter ${ }^{26}$ (Supplementary Fig. 2a). We created a 128 second dataset $\mathbf{Y}$ and made it identical to $\mathbf{X}: \mathbf{X}=\mathbf{Y}$. We then constructed a gene score matrix $\mathbf{Z}$ 129 from $\mathbf{Y}$ by permuting a fraction of features (rows), termed misalignment rate (MR), into different 130 orders. The features between $\mathbf{Z}$ and $\mathbf{Y}$ are perfectly aligned if MR equals 1 and are independent if 131 MR equals 0 . We further added white noise on all the entries of $\mathbf{Z}$ at a given signal-noise-ratio 132 (SNR) level. 
134 We then provide (X, Z) as input to the other methods (Supplementary Fig. 2b), mimicking how 135 they perform integration, while provide both $(\mathbf{X}, \mathbf{Z})$ and $(\mathbf{Y}, \mathbf{Z})$ to bindSC (Supplementary Fig. 136 2c). As described, rather than taking $\mathbf{Z}$ as it is from the input, bindSC will iteratively update $\mathbf{Z}$ 137 until reaching convergence.

139 Since we know the true cell type and dataset origin of the cells in these experiments, we can assess

140 the integration performance in terms of cell type classification accuracy and dataset alignment

141 accuracy in the co-embeddings. It is necessary to measure both types of accuracy, as a high cell 142 type classification accuracy can be achieved by simply projecting cells onto local clusters without 143 achieving uniform mixing of the two datasets. Similarly, a high dataset alignment accuracy can be 144 achieved by uniformly mixing cells from the datasets, regardless of their cellular identity. We used 145 Silhouette score for measure cell type classification accuracy and alignment mixing score to 146 measure the dataset alignment accuracy (Methods). We compared bindSC, CCA, Seurat, LIGER 147 and Harmony under default settings (Supplementary Note 1).

149 We obtained results from a range of MRs under SNR $=0.25$ (Fig. 2). When there was no feature 150 misalignment $(\mathrm{MR}=0)$, all methods achieved good performance. Even under this ideal scenario, 151 bindSC achieved the highest Silhouette score (>0.75) (Fig. 2a). The worse performance of other 152 methods can be explained by the noise introduced to distort the manifold structures between $\mathbf{X}$ and

153 Z. CCA showed better performance than Seurat, which may be due partly to label transferring 154 errors introduced by Seurat's empirical anchor-based alignment approach. As MR increased from 1550 to 0.9 , the Silhouette score for bindSC remained stable $(>0.7)$, while all the other methods 156 showed a decreasing trend, especially for LIGER and Harmony. Harmony worked well when MR 
$157 \leq 0.15$ (Fig. 2a-b) but had a substantial drop on Silhouette score $(<0.1)$ when $\mathrm{MR}>0.15$. In

158 addition, its alignment mixing score dropped to 0 when $\mathrm{MR}>0.2$, with no mixing of cells from $\mathbf{X}$

159 and $\mathbf{Z}$ in the co-embedding UMAP (Fig. $2 \mathbf{b} ; \mathrm{MR}=0.5$ ). Harmony takes cell coordinates from a

160 reduced dimensional PCA space and runs an iterative algorithm to adjust for dataset-specific

161 effects. When $\mathrm{MR}>0.15$, cells from $\mathbf{X}$ and $\mathbf{Z}$ already formed two dis-joint groups, which made

162 the downstream integration impossible for Harmony. The Silhouette score of LIGER showed

163 fluctuations but was always lower than 0.4. LIGER utilizes an integrated nonnegative matrix

164 factorization (iNMF) method to identify shared and dataset-specific metagenes across two datasets.

165 If it worked as designed, the errors caused by feature misalignment should be contained within

166 dataset-specific modules. However, variance explained by the data-specific modules appeared to

167 be small $(<1 \%)$. When $\mathrm{MR} \geq 0.95$, all methods including bindSC failed to achieve reasonable

168 integration. That was expected as $\mathbf{X}$ and $\mathbf{Z}$ (as well as $\mathbf{Y}$ and $\mathbf{Z}$ ) became nearly independent.

170 As expected, increasing SNR level worsened the integration performance for most of the methods

171 except bindSC. For example, both CCA and Seurat had acceptable performance under MR $=0.5$

172 and SNR $=0$ (Supplementary Fig. 3a), but Seurat failed to separate cell type 2 and 3 accurately

173 when $\mathrm{SNR}=0.25$ or 0.5 (Supplemental Fig. 4a; Fig. 2). For SNR = 0.5, Harmony failed in both

174 alignment mixing $(<0.2)$ and classification $(=0)$ accuracy, even when MR was as low as 0.1

175 (Supplementary Fig. 4).

177 We repeated the above experiments by increasing the number of cells to 5,000 and 10,000, 178 respectively. Similarly, bindSC showed robust performance regardless of MR and SNR levels, 179 which was not achieved by other methods (Supplementary Tables S2-3). Overall, the simulation 
180 results demonstrated that bindSC is robust to bias introduced by noise in the data and via pre-

181 aligning features, thanks to its ability to align both cells and features simultaneously.

183 Integrating single cell epigenomic data with single cell transcriptomic data

184 Integrating single cell epigenomic data with single cell transcriptomic data obtained from

185 unimodal technologies provides an opportunity to decipher epigenetic regulatory mechanisms

186 underpinning cell transcriptomic identity. We examined the performance of bindSC in integrating

187 the scRNA-seq and scATAC-seq data derived from lung adenocarcinoma (A549) cells after 0, 1,

188 and 3 hours of dexamethasone (DEX) treatment ${ }^{6}$. This dataset was generated using a

189 combinatorial indexing-based coassay (sci-CAR), which enabled jointly measurement of

190 chromatin accessibility and transcriptome in the same cells. In this dataset, 6,005 cells have sci-

191 RNA-seq profiles and 3,628 cells have sci-ATAC-seq profiles. Among them, 1,429 cells have both

192 RNA-seq and ATAC-seq profiles, which can be used as a gold standard for evaluating integration

193 accuracy of various methods (Methods).

195 For comparison, we ran the 4 methods on the same data and derived in silico co-embeddings. There

196 was relatively clear separation between cells acquired at 0 hour and those at 1 or 3 hours in the co-

197 embeddings (Fig. 3a). In terms of classifying cells by time, bindSC achieved the highest Silhouette

198 score and Harmony the second, whereas Seurat had the lowest score with many sub-clusters in its

199 co-embedding (Fig. 3a-b). As to alignment accuracy, bindSC and Harmony had similar scores,

200 whereas Seurat received a relatively low score (Fig. 3b). Similar trends were observed in a

201 previous study analyzing the same dataset ${ }^{27}$. As suggested by simulation, the low alignment

202 mixing score of Seurat was likely attributable to bias introduced in its anchor-based integration. 
204 A perfect integration method would place the two instances of the 1,429 co-assayed cells onto 205 identical locations in the co-embeddings. We leveraged this expectation to compare the accuracy 206 of various methods. We defined a metric, called anchoring distance that measures the normalized 207 Euclidean distance between the two instances of a co-assayed cell in the co-embeddings 208 (Methods). BindSC achieved substantially shorter anchoring distances than the other methods ( $p$ $209<2.2 \mathrm{e}-16$; Student t-test; Fig. 3b).

211 We further compared how accurately TF (or peak) -gene correlations can be inferred from the co212 embeddings produced by each method. For a fair comparison, we applied the same bindSC 213 workflow to derive pseudo-cells for the 4 methods (Methods; Supplementary Note 2).

215 For each TF-gene (and peak-gene) pair, we calculated a Spearman rank correlation coefficient 216 (SRCC) between the TF activity (and normalized peak) level and the gene expression level in the 217 pseudo-cells (Methods). We repeated the same calculation in the co-assayed cells to create a gold 218 standard. For each of the 4 methods in 3 types of relations: TF-gene, cis- peak-gene and trans219 peak-gene, we calculated a summary SRCC between the SRCCs obtained from the pseudo-cells 220 and the SRCCs obtained from the co-assayed cells. The summary SRCCs resulting from bindSC 221 were consistently higher than those obtained from Seurat, LIGER and Harmony in all the 222 categories of comparison, indicating that the bindSC multiomic profile had the highest accuracy.

224 We further examined the peak-gene association identified from the co-assayed cell profiles and 225 found 585 trans- peak-gene pairs being supported by isogenic Hi-C data generated in an 
226 independent study ${ }^{28}$. Compared with other approaches, bindSC derived peak-gene SRCCs of the

227 highest level of agreement with those observed in the co-assayed cells (Supplementary Fig. 5).

228 Among the 585 trans- peaks, 470 appeared more strongly correlated with the corresponding gene

229 expression levels than did the corresponding cis- peaks. One example was the gene CFLAR and a

230 trans- peak at chr2:201,770,437-201,770,992, which is 200-kb upstream of CFLAR transcription

231 start site, spanning over three genes (Fig. 3e). The SRCC of this pair was 0.32 in the co-assayed

232 cells. It was lower but comparable (0.23) in the bindSC pseudo-cells, however, became

233 substantially lower $(<0.11)$ in the pseudo-cells generated by the other methods (Supplementary

234 Fig. 5).

236 The DEX treatment specifically targets the glucocorticoid receptor encoded by NR3C1, a TF that

237 activates the mRNA transcription of a handful of downstream genes. BindSC accurately

238 reconstructed the gene expression and TF activity kinetics of NR3C1 (Fig. 3d), consistent with

239 what was depicted in the original study ${ }^{6}$ using the co-assayed cells: the $N R 3 C 1$ expression level

240 decreased over time while its activity level increased; Even the slowing down trend of NR3C1

241 activity was captured.

243 We further evaluated the performance of bindSC in integrating scRNA-seq and scATAC-seq on

244 another available multi-omics dataset generated recently by SHARE-seq technology ${ }^{14}$. There were 245 a total of 37,774 cells from mouse skin tissues that had paired RNA and ATAC profiles. Compared 246 with other methods, bindSC again achieved significantly shorter anchoring distances

247 (Supplementary Fig. 7; Supplementary Note 4). 


\section{Comprehensive evaluation using a novel mouse retinal cell atlas}

250 For comprehensive evaluation and comparison, we generated a novel multi-omics dataset from

251 single nuclei of wild type mouse retina. Mouse retina is heterogeneous, composed of multiple

252 neuronal and non-neuronal cell types, including five major neuron classes: photoreceptors (rods

253 and cones), retinal ganglion cells (RGC), horizontal cells (HC), bipolar cells (BC), amacrine cells

254 (AC), and a non-neuronal Müller glial cell (MG) ${ }^{4,29,30}$. While we ${ }^{29}$ and others ${ }^{31-33}$ have provided

255 high-resolution single cell transcriptomic profiles of whole retina or specially sorted cell types on

256 mouse and human retina tissue, little is known on the single-cell chromatin landscape of mouse

257 retina tissue. Numerous studies ${ }^{34-36}$ demonstrate the importance of transcription factors (TFs) on

258 establishing or maintaining the chromatin landscapes that define retina cell identity. Therefore,

259 integration of ATAC and RNA profiles at single cell resolution provides an exciting opportunity

260 to comprehensively characterize cell types and rare cell subtypes in mouse retina.

262 We applied the newly released 10x Genomics Multiome ATAC+RNA kit on nuclei suspension

263 acquired from adult mice retina samples. After performing standard quality control, we obtained

264 an atlas of 9,383 nuclei of high-quality ATAC+RNA profiles. To define cell types, we first

265 clustered the RNA and the ATAC data individually. Nineteen (19) clusters were identified from

266 the RNA data alone, which included all the known major cell types with some subtypes identified:

267 rod, $\mathrm{BC}(\mathrm{BC} 1 \sim \mathrm{BC} 10), \mathrm{AC}, \mathrm{RGC}$, cone, $\mathrm{HC}, \mathrm{MG}$ and retina progenitor cells (RPC) (Fig 4a and

268 Supplementary Fig. 8). Nineteen (19) clusters were also identified from the peak files of the

269 ATAC data alone (Fig. 4b). Although known cell types appeared to be well separated in both

270 modalities, there were some noticeable differences. For example, RGC cells and rod cells were

271 separated clearly in the RNA data but partly blended together in the ATAC data, whereas ACs and 
272 RGC cells were blended in the RNA data but well separated in the ATAC data. Interestingly, all

273 the $10 \mathrm{BC}$ cell subtypes, defined based on RNA expression levels, were well separated in the

274 ATAC data except for BC1 and BC6. However, after reducing ATAC data to gene level in a gene

275 activity matrix, the cell types became considerably harder to delineate (Fig. 4c).

277 To obtain in silico multiomics profiles, we ran bindSC together with three other methods on the

278 data without using the known cell correspondence. As shown in the co-embedding UMAP

279 (Fig. 4d-e), bindSC successfully aligned cell types across modalities, with most cell types well

280 separated out (Fig. 4d-f). Interestingly, bindSC successfully aligned the HCs, which is quite rare

281 in the dataset ( 23 cells, $<0.25 \%$ abundance). None of the other methods aligned the HCs correctly

282 as it was already difficult to separate the HCs from the ACs in the gene-level chromatin profiles

283 (Fig. 4c and Supplementary Fig. 9), the input to the other methods. Overall, the anchoring

284 distances in the co-embeddings generated by bindSC were considerably smaller than those

285 generated by the other methods in all the cell types assessed (Fig. 4f).

287 Note that bindSC aligned the 10 BC subtypes reasonably well (Fig. 4g), although separations in 288 the ATAC modality were not as clean as they were in the RNA modality. In comparison, Seurat 289 and LIGER failed to generate meaningful alignments among the BC subtypes (Fig. $\mathbf{4 h}-\mathbf{j}$ and 290 Supplementary Fig. 9) while Harmony aligned a few subtypes successfully. These were due 291 partly to the fact that these methods used the low precision gene-level chromatin accessibility 292 profiles as the input (Fig. 4c). 
294 Overall, our study demonstrated the power of multiomics in delineating rare cell types and proves

295 that bindSC can generate in silico multiomics profiles that are considerably more accurate than do

296 existing tools.

297

298 Integrating scRNA-seq data with spatial transcriptomics (ST) data

299 BindSC can integrate scRNA-seq data with spatial transcriptomics data to 1) assign spatial

300 locations to cells in the scRNA-seq data and 2) associate additional RNA features to the spatial

301 data for higher resolution delineation. For demonstration, we applied bindSC to integrate the

302 SMART-Seq2 data with the in situ spatial transcriptomics data generated by 10x Visium from the

303 same mouse frontal cortex tissue. These two datasets differ widely in number of cells: 1,072 spots

304 in the ST data versus 14,249 cells in the scRNA-seq data (Supplementary Fig. 10a). The spots

305 on the Visium assay are at $\sim 50$ um resolution and each spot can contain tens of cells. There were

3066 clusters identified from the ST data alone, which linked to distinct layers in the corresponding

307 histology images (Supplementary Fig. 10b-c) and 23 cell types from the scRNA-seq data alone

308 (Supplementary Fig. 10d).

310 We used bindSC and other programs to derive co-embeddings containing datapoints from both

311 datasets (Fig. 5a). BindSC achieved evidently higher alignment mixing scores than the other

312 programs (Supplementary Fig. 11c) while the Silhouette scores were similar (Supplementary

313 Fig. 11b). For each pseudo-cell in the scRNA-seq data, we calculated its probability to map to a

314 spatial location in the histology image. We then overlaid these cells on the histology image

315 coloring by their probability scores (Methods). Noticeably, several cell types in the scRNA-seq

316 data mapped to distinct spatial layers in the histology image, which is consistent with the known 
317 cellular anatomy of mouse cortex, particularly for the laminar excitatory neuron cell types such as

318 L2.3 IT, L4, L5.IT, L5.PT, L6.IT, L6.CT, L6B and NP (Fig. 5b). Consistent with previous

319 observations, the oligodendrocyte-rich white matter (oligo cells) was mapped below the cortex.

320 BindSC and Seurat were also able to map inhibitory clear cell types such as Lamp5, Vip, Pvalb

321 and Sst in the scRNA-seq data to the histology image, but these cell types did not form distinct

322 spatial patterns. LIGER and Harmony, which had worse alignment mixing scores (Supplementary

323 Fig. 11c), failed to map these cells (Supplementary Figs. 13-14), especially the Vip cells. The

324 poor mapping of the inhibitory cells may also be attributable to the limited resolution of the Visium

325 technology.

327 Given that each spot in the ST data may encompass multiple cells from multiple cell types, we

328 hypothesized that the probability scores calculated from the co-embeddings can reveal the

329 composition of the cell types at each spot. Fig. 5c showed the relationship between cell type

330 abundance in the scRNA-seq data and abundance estimated based on probability scores in the ST

331 data. Results from bindSC achieved the best correlation (Pearson's R = 0.9). L6.IT, Sst and Vip

332 cell types were the top 3 most abundant cell types in both the scRNA-seq data and the ST data.

333 Seurat also performed reasonably well (Pearson's $\mathrm{R}=0.83$ ) while LIGER and Harmony performed

334 worse. Note that Lamp5 was the cell type that showed the largest discordance in the bindSC result.

335 In examining the spatial distributions of Lamp5 specific gene expressions such as Lsp1, Npy2r,

336 and Dock5, we could not find any spatial patterns (Supplementary Fig. 11 d-e). This finding may

337 indicate that Lamp5 does not have a characteristic spatial distribution. 
340 Complex interplay exists between mRNAs and proteins ${ }^{37}$. Single-cell proteomic methods such as

341 mass cytometry (CyTOF) ${ }^{2,38}$ measure abundance of a small set of (often 10-50) surface proteins

342 (epitopes) and provide functional quantification of various cell populations. Integrating single-cell

343 RNA and protein data from the same sample can potentially achieve higher resolution

344 characterization and enable discovery of novel cellular states and associated features. BindSC can

345 be applied for such a task. Notice that this task cannot be achieved by any of the existing tools

346 because the mRNA and protein expression levels derived from the same genes are not well

347 correlated, due to complex post-transcriptional modifications and technological limitations ${ }^{39}$.

348 CITE-seq ${ }^{40}$ performs jointly profiling of epitope and mRNA levels in the same cells and can be

349 used to evaluate the results of in silico integration.

351 We used a CITE-seq dataset consisting of 30,672 human bone marrow cells with a panel of 25

352 antibodies ${ }^{17}$. We split the data into an RNA matrix and a protein matrix. Unsupervised clustering

353 of the RNA matrix revealed cell types largely consistent with those in the protein matrix, except

354 for some noticeable differences (Fig. 6a-b). CD8+ and CD4+ T cells were partly blended together

355 in the RNA data but separated clearly in the protein data. On the other hand, conventional dendritic

356 cells (cDC2) were separated from other clusters in the RNA data but were intermixed with other

357 cell types in the protein data. In contrast, unsupervised clustering of the gene expression levels of

358 the 25 protein-homologous RNAs could not yield meaningful classification (Fig. 6c).

359 Consequently, Seurat, LIGER and Harmony, which work with only data matrix of 25 homologous

360 features, failed to produce meaningful co-embeddings (Supplementary Fig. 15): the cells from

361 the protein data were well clustered, but those from the RNA data were not meaningfully

362 distributed in the co-embeddings. 
364 We then tested bindSC on this task. The matrix $\mathbf{X}$ was set as the protein matrix, $\mathbf{Y}$ the RNA matrix

365 of 3,000 highly variable genes, and $\mathbf{Z}$ the RNA matrix containing only the 25 protein-homologous

366 genes. Remarkably, the majority of the cells from the two modalities became well aligned in the

367 co-embedding (Fig. 6d-e), as they are expected to be. Similar to our previous experiments, we

368 calculated the anchoring distance between the protein and the RNA cells deriving from the same

369 original cells in the co-embeddings. The overall anchoring distance for bindSC was significantly

370 lower than those obtained by Seurat, LIGER, Harmony, or random guesses (p-value $<2.2 \mathrm{e}-16$;

371 Student t-test; Fig. 6f). Notably, the bulk of CD4+ and CD8+ T cells in the RNA data became well

372 separated in the co-embedding (Fig. 6d-e), thanks to the power of integration. Moreover, the

373 anchoring distances revealed the extent of differences between the levels of the RNAs and those

374 of the homologous proteins in individual cell types (Fig. 6g). Interestingly, relatively rare cell

375 types such as HSC, Prog/NK, LMPP, and CD16+ Mono appeared relatively well anchored,

376 whereas relatively common cell types such as CD8 naïve, CD8 memory, B progenitor, Treg, etc.

377 appeared less well anchored. This indicates that there are higher degrees of post-transcriptional

378 heterogeneity in cell types conducting adaptive immune surveillance ${ }^{41}$.

380 Discussion

381 Despite the ground-breaking advances in single-cell technologies, including multiomics

382 technologies, there always exists a need to computationally integrate multiple data matrices of

383 different modalities from the same biological samples to derive a more comprehensive 384 characterization of cellular identities and functions. 
386 Our method bi-CCA and tool bindSC appeared to have addressed this important analytical

387 challenge without compromising biological complexity in the data. In our experiments, bindSC

388 successfully integrated data obtained from a wide variety of vastly different technologies covering

389 transcriptomes, epigenomes, spatial-transcriptomes and proteomes, and clearly outperformed

390 existing tools such as Seurat, LIGER and Harmony, when being evaluated objectively using true

391 single-cell multiomics data derived from the same cells. In particular, Seurat, LIGER, and

392 Harmony are essentially first-order solutions that can be applied to only rows or columns but not

393 both simultaneously. That approach introduced biases in the results and restricted the utility of

394 those tools in discovering complex cell-type relations and molecular interactions. For instance,

395 they consider only the basic cis-regulatory relations and ignores trans-regulatory relations 6

396 established via distal enhancers, as exemplified in the interaction between CFLAR and a $200 \mathrm{kbps}$

397 upstream putative enhancer site discovered by bindSC and validated by Hi-C in the DEX-treated

398 A549 data. Other scATAC-seq analysis pipelines such as MAESTRO ${ }^{16}$ and ArchR ${ }^{42}$ have similar

399 restrictions.

400

401 Similarly, bindSC was able to meaningfully associate the expression levels of mRNAs with those

402 of the surface proteins, a very challenging task due to complexity in post-transcriptional

403 modification. The resulting co-embedding offered deeper biological insights than embeddings

404 derived from single modality or by using other existing approaches. For example, CD4+ T cells

405 became evidently separated from CD8+ T cells and so did pDC cells from other cell types. 
407 BindSC also achieved meaningful mapping of scRNA-seq data to spatial locations in the brain 408 cortex samples, after integrating with the ST data. Even though the two datasets were not both at 409 single-cell resolution, bindSC was still able to achieve a meaningful integration.

411 Bi-CCA made two assumptions: 1) the two sets of cells are sampled uniformly from the same

412 biological sample; 2) the features of the two datasets are linearly correlated. These two 413 assumptions are met under many scenarios of current investigations, however, could be violated 414 when there are insufficient number of cells obtained from a rapidly developing cell population.

415 Consequently, the accuracy of the co-embedding could vary, depending on the sampling density 416 and the complexity of the population. We measured accuracy with respect to data complexity in 417 the simulation experiments, however, accuracy on a real dataset could be complex to gauge $a$ 418 priori and will require case by case investigation in the context of a specific study, followed by 419 necessary experimental validation. Nonetheless, in this study we clearly proved based on objective 420 ground truth data that bi-CCA substantially avoided bias introduced by existing methods and that 421 bindSC is a robust implementation that can be applied to derive meaningful results on most recent 422 datasets containing thousands to tens of thousands cells (Supplementary Table 1).

424 BindSC is efficiently implemented in $\mathrm{R}$. The major computational cost for bindSC is from 425 calculating cell/feature co-embedding coordinates using singular value decomposition (SVD) 426 (Methods); It typically requires $O(M N L)$ floating-point operations to construct $M N$ cell-cell 427 distance matrix as input to SVD decomposition, where $M$ and $N$ are cell number of the two 428 modalities, respectively. To address this computational challenge, bindSC implements the "divide429 and-conquer eigenvalue algorithm”. The divide part first splits cells into different blocks specified 
430 by users, which can be solved in parallel with lower memory usage (Supplementary Fig. 1b). The

431 conquer part then merges results from each block recursively. Therefore, the maximal memory

432 usage of bindSC is independent of the total cell number.

433

434 Taken together, we believe that bindSC is likely the first tool that has achieved unbiased integration

435 of data matrices generated by different technologies and can be applied in broad settings. In the 436 single-cell domain, bindSC can clearly be applied to align cells and features simultaneously, which

437 are important for ongoing investigations in the Human Cell Atlas ${ }^{43}$, the NIH HubMap ${ }^{44}$, the 438 Human Tumor Cell Network ${ }^{45}$ and on remodeling of tumor microenvironment ${ }^{46}$. Further, bindSC

439 can potentially be applied to other domains, such as integrating patient sample mRNA profiles

440 with cell-line drug-sensitivity data ${ }^{47}$. 


\section{$441 \quad$ Methods}

\section{BindSC workflow}

443 BindSC workflow for creating in silico single cell multi-omics embeddings consists of five steps:

444 1. individual dataset preprocessing including variable feature selection and cell clustering,

2. initializing feature matching across modalities (i.e., constructing gene score matrix),

3. identifying cell correspondence using the bi-CCA algorithm,

4. jointly clustering cells between two modalities in the co-embedding latent space and constructing pseudo-cell level multi-omics profiles, and

450 We formulate our method for the case of two modalities. Let $\boldsymbol{X} \in \mathbb{R}^{M \times K}$ be a single-cell dataset of 451 features $g_{1}, g_{1}, \cdots, g_{M}$ by cells $c_{1}, c_{1}, \cdots, c_{K}$ and $\boldsymbol{Y} \in \mathbb{R}^{N \times L}$ be a single-cell dataset of feature $452 p_{1}, p_{2}, \cdots, p_{N}$ by cells $d_{1}, d_{1}, \cdots, d_{L} . M$ and $N$ are the numbers of features (e.g., gene expression, 453 chromatin accessibility, protein abundance level) in the two datasets. $K$ and $L$ are the number of 454 cells in the two datasets. Without loss of generality, we assume that features $455 g_{1}, g_{1}, \cdots, g_{M}$ represent the gene expression levels and $M \leq N$. The important component of each 456 step is described as follows.

\section{Individual modality preprocessing}

459 For each modality, we follow standard processing pipeline, which includes variable feature 460 selection and unsupervised cell clustering. The cluster information derived from each modality is 461 used for downstream parameter optimization.

\section{Initializing feature matching across modalities}


464 Because features in the two datasets are generally different, bindSC requires one additional

465 transition matrix $\boldsymbol{Z} \in \mathbb{R}^{M \times L}$ as input for bridging the integration of $\boldsymbol{X}$ and $\boldsymbol{Y}$. The transition matrix

$466 \boldsymbol{Z}$ can be understood as the projection of $\boldsymbol{Y}$ to the feature space of the first dataset $\boldsymbol{X}$. Taking the

467 integration of scRNA-seq and scATAC-seq as an example, the matrix $\boldsymbol{Z}$ can be derived from

468 scATAC-seq profiles by summing reads in gene bodies ${ }^{17,19,23}$. This can also be input from the

469 regulatory potential (RP) model in MAESTRO ${ }^{16}$. In a simpler case where $\boldsymbol{X}$ and $\boldsymbol{Y}$ have matched

470 features, the integration tasks fall into two categories: 1) batch correction for scRNA-seq data

471 across individuals, species, or technologies; 2) integration of scRNA-seq with spatial

472 transcriptome data. In those cases, the transition matrix $\boldsymbol{Z}$ is initialized as $\boldsymbol{Y}$. In bi-CCA, $\boldsymbol{Z}$ is

473 updated iteratively. In the following text, the initial value of $\boldsymbol{Z}$ is denoted by $\boldsymbol{Z}^{(\mathbf{0})}$.

\section{Bi-order canonical correlation analysis (Bi-CCA)}

476 The key algorithm implemented in bindSC is Bi-CCA, the concept of which extends traditional $477 \mathrm{CCA}^{17,24,48}$ to both rows and columns to enable capturing of correlated variables in cells and 478 features simultaneously. Bi-CCA introduces two cell-level projection matrices $\boldsymbol{U} \in \mathbb{R}^{K \times E}, \boldsymbol{S} \in$ $479 \mathbb{R}^{L \times E}$ such that the correlations between indices $\boldsymbol{X} \boldsymbol{U}$ and $\boldsymbol{Z S}$ are maximized, and two feature-level 480 projection matrices $\boldsymbol{T} \in \mathbb{R}^{M \times E}, \boldsymbol{V} \in \mathbb{R}^{N \times E}$ such that the correlations between indices $\boldsymbol{Z}^{\prime} \boldsymbol{T}$ and $\boldsymbol{Y}^{\prime} \boldsymbol{V}$ 481 are maximized. The optimization framework can be formulated as:

$$
\underset{\boldsymbol{U}, \boldsymbol{S}, \boldsymbol{T}, \boldsymbol{V}, \boldsymbol{Z}}{\operatorname{argmax}} \operatorname{tr}\left\{(\boldsymbol{X U})^{\prime} \boldsymbol{Z S}+\left(\boldsymbol{Z}^{\prime} \boldsymbol{T}\right)^{\prime} \boldsymbol{Y}^{\prime} \boldsymbol{V}\right\}
$$

$$
\text { subject to }(\boldsymbol{X U})^{\prime} \boldsymbol{X U}=\boldsymbol{I},(\boldsymbol{Z S})^{\prime} \boldsymbol{Z S}=\boldsymbol{I},\left(\boldsymbol{Z}^{\prime} \boldsymbol{T}\right)^{\prime} \boldsymbol{Z}^{\prime} \boldsymbol{T}=\boldsymbol{I}, \quad\left(\boldsymbol{Y}^{\prime} \boldsymbol{V}\right)^{\prime} \boldsymbol{Y}^{\prime} \boldsymbol{V}=\boldsymbol{I} \text {. }
$$

485 If the transition matrix $\boldsymbol{Z}$ is known, the objective (1) can be divided into two disjoint traditional 486 canonical correlation analysis (CCA) problems. The left term is performed to identify cells of 
487 similar (aligned) features, while the right term is performed to identify features shared by the 488 (aligned) cells, each of which can be solved in the CCA framework. However, it is difficult to 489 update transition matrix $\boldsymbol{Z}$ in equation (1) even when matrices $\boldsymbol{U}, \boldsymbol{S}, \boldsymbol{T}, \boldsymbol{V}$ are available. This is 490 because: a) left optimization problem requires $\boldsymbol{Z}$ as input and the right optimization problem 491 requires $\boldsymbol{Z}^{\prime}$ as input, leading (1) to a non-linear optimization problem; b) transition matrix $\boldsymbol{Z}$ shows 492 up in constraints.

494 Therefore, we modify equation (1) in a much more practical way. First, we standardize $\boldsymbol{X}$ to let it 495 have $\boldsymbol{X}^{\prime} \boldsymbol{X}=\boldsymbol{I}$, and standardize $\boldsymbol{Y}$ so that $\boldsymbol{Y} \boldsymbol{Y}^{\prime}=\boldsymbol{I}$. The standardization process can be seen in 496 Algorithm 1. Thus, equation (1) could be simplified as and $\boldsymbol{Z}_{r} \in \mathbb{R}^{M \times L}$ and optimize the following problem:

$$
\underset{\boldsymbol{U}, S, T, V, Z_{l}, Z_{r}}{\operatorname{argmax}} \operatorname{tr}\left\{(\boldsymbol{X U})^{\prime} \boldsymbol{Z}_{l} \boldsymbol{S}+\left(\boldsymbol{Z}_{r}^{\prime} \boldsymbol{T}\right)^{\prime} \boldsymbol{Y}^{\prime} \boldsymbol{V}\right\}+\left\|\boldsymbol{Z}_{l}-\boldsymbol{Z}_{r}\right\|_{2}
$$

505 To solve equation (3), we also standardize $\boldsymbol{Z}^{(\mathbf{0})}$ to let $\boldsymbol{Z}^{(\mathbf{0})^{\prime}} \boldsymbol{Z}^{(\mathbf{0})}=\boldsymbol{I}$, and initialized with $\boldsymbol{Z}_{l}$ : $=\boldsymbol{Z}^{(\mathbf{0})}$.

506 The standard singular value decomposition (SVD) can be implemented to obtain the canonical

507 correlation vectors $(\mathrm{CCVs})$ at cell levels. We used a user-defined number $(E)$ of singular vectors

508 that approximate the CCVs (Algorithm 2). Here we term $E$ to represent the cell-level 
509 "dimensionality" in the latent space, which is a parameter required to be optimized (Details seen

510 in Parameter optimization).

$$
(\boldsymbol{U}, \boldsymbol{S}):=\underset{\boldsymbol{U}, \boldsymbol{S}}{\operatorname{argmax}} \operatorname{tr}\left(\boldsymbol{U}^{\prime} \boldsymbol{X}^{\prime} \boldsymbol{Z}_{l} \boldsymbol{S}\right) \text { subject to } \boldsymbol{U}^{\prime} \boldsymbol{U}=\boldsymbol{I}, \boldsymbol{S}^{\prime} \boldsymbol{S}=\boldsymbol{I} .
$$

513 Having CCV pair $(\boldsymbol{U}, \boldsymbol{S})$ obtained, we have cell correspondence in the latent space between two

514 datasets. The left transition matrix $\boldsymbol{Z}_{l}$ can be updated by:

$$
\left(\boldsymbol{Z}_{l}\right):=\underset{\boldsymbol{Z}_{l}}{\operatorname{argmax}} \operatorname{tr}\left(\boldsymbol{U}^{\prime} \boldsymbol{X}^{\prime} \boldsymbol{Z}_{l} \boldsymbol{S}\right) \text { subject to } \boldsymbol{Z}_{l} \boldsymbol{Z}_{l}^{\prime}=\boldsymbol{I} \text {. }
$$

517 The details of solving optimization problem (5) is in Algorithm 2.

519 We then set

$$
\left(\boldsymbol{Z}_{r}\right):=\boldsymbol{Z}_{l}
$$

521 The similar SVD algorithm (Algorithm 2) is used to approximate CCVs:

$$
(\boldsymbol{T}, \boldsymbol{V}):=\underset{\boldsymbol{T}, \boldsymbol{V}}{\operatorname{argmax}} \operatorname{tr}\left(\boldsymbol{T}^{\prime} \boldsymbol{Z}_{r} \boldsymbol{Y}^{\prime} \boldsymbol{V}\right) \text { subject to } \boldsymbol{T}^{\prime} \boldsymbol{T}=\boldsymbol{I}, \boldsymbol{V}^{\prime} \boldsymbol{V}=\boldsymbol{I} .
$$

$$
\left(\boldsymbol{Z}_{r}\right):=\underset{Z_{r}}{\operatorname{argmax}} \boldsymbol{t r}\left\{\left(\boldsymbol{Z}_{r}^{\prime} \boldsymbol{T}\right)^{\prime} \boldsymbol{Y}^{\prime} \boldsymbol{V}\right\} \text { subset to } \boldsymbol{Z}_{r}^{\prime} \boldsymbol{Z}_{\boldsymbol{r}}=\boldsymbol{I}
$$

529 Next, we set

$$
\left(\boldsymbol{Z}_{l}\right):=\boldsymbol{Z}_{r}
$$


531 The update process (4) (9) are repeated until convergence. Because each of the subproblems is 532 convex with respect to the block variables being optimized, the algorithm is guaranteed to 533 converge to a fixed point (local minimum).

535 In the above framework, the transition matrix $\boldsymbol{Z}$ (represented by $\boldsymbol{Z}_{l}$ and $\boldsymbol{Z}_{r}$ ) is updated based on 536 original observed matrices $\boldsymbol{X}$ and $\boldsymbol{Y}$. In practice, we introduce the couple coefficient $\alpha(0 \leq \alpha \leq 1)$ 537 to assign weights on initialized matrix $\boldsymbol{Z}^{(\mathbf{0})}$ on transition process (6) and (9).

$$
\left(\boldsymbol{Z}_{r}\right):=(1-\alpha) \boldsymbol{Z}^{(\mathbf{0})}+\alpha \mathbf{Z}_{l}
$$

539 and

$$
\left(\boldsymbol{Z}_{l}\right):=(1-\alpha) \boldsymbol{Z}^{(\mathbf{0})}+\alpha \mathbf{Z}_{r}
$$

541 The couple coefficient $\alpha$ can reflect the contribution of initial $\boldsymbol{Z}^{(\mathbf{0})}$ on linking two modalities.

542 Equations (10) and (11) will be reduced to Equations (6) and (9) if $\alpha=1$. The bi-CCA algorithm

543 will be reduced to traditional CCA if $\alpha=0$. Selection of coefficient $\alpha$ can be seen in Parameter

544 optimization. Notably, the final $\boldsymbol{Z}_{r}$ and $\boldsymbol{Z}_{l}$ will be converged to different matrices if $\alpha<1$. The 545 workflow of the iterative process is shown in Supplementary Fig. 1a.

547 Jointly clustering cells across datasets in shared latent space and constructing pseudo-cell level multi-omics profiles

549 Equation (4) projects cells of two datasets into a correlated $E$-dimensional space with cell 550 coordinates $\boldsymbol{U}=\left(\boldsymbol{u}_{1}, \boldsymbol{u}_{2}, \ldots, \boldsymbol{u}_{K}\right)$ and $\boldsymbol{S}=\left(\boldsymbol{s}_{1}, \boldsymbol{s}_{2}, \ldots, \boldsymbol{s}_{L}\right)$, respectively. L2-normalization is 551 performed to remove global differences in scale, therefore

$$
\widehat{\boldsymbol{u}}_{i}=\boldsymbol{u}_{i} /\left\|\boldsymbol{u}_{i}\right\|_{2}, i=1,2, \ldots, K
$$

$$
\hat{\boldsymbol{s}}_{i}=\boldsymbol{s}_{i} /\left\|\boldsymbol{s}_{i}\right\|_{2}, i=1,2, \ldots, L
$$


555 The shared nearest neighbor ( $\mathrm{SNN})$ graph is constructed by calculating the $l$-nearest neighbors $(20$

556 by default) based on the Euclidean distance of L2-normlized latent space. The fraction of shared 557 nearest neighbors between the cell and its neighbors is used as weights of the SNN graph. The 558 modularity optimization technique Leiden algorithm ${ }^{49}$ is used to group cells into interconnected 559 clusters (termed meta-cluster) based on constructed SNN graph with a resolution parameter setting 560 by users (default 0.5 ).

562 To understand the molecular-level interaction among modalities, we construct the pseudo-cell

563 level multi-omics profiles. Briefly, for cells in each meta-cluster identified, the Leiden algorithm

564 is further performed based on SNN graph with a higher resolution (default $=2)$. In this way, cells

565 in each meta-cluster are further grouped into highly interconnected sub-clusters. We term such

566 sub-clusters as pseudo-cells. Only pseudo-cells that consist of at least $n$ cells (default $=10)$ are

567 kept for downstream analysis, while the others are considered data-specific and discarded. Profiles

568 of the pseudo-cells are constructed by aggregating the cells included. We denote by $\boldsymbol{X}^{\text {pseudo }} \in$ $569 \mathbb{R}^{M \times P}$ be pseudo-cell profiles of feature $g_{1}, g_{1}, \cdots, g_{M}$ and $\boldsymbol{Y}^{\text {pseudo }} \in \mathbb{R}^{N \times P}$ be pseudo-cell profiles 570 of feature $p_{1}, p_{2}, \cdots, p_{N} . P$ is the number of pseudo-cells.

\section{Algorithm 1. Standardizing inputs}

573 For input matrix $\boldsymbol{X}$, we denote $\boldsymbol{\Sigma}_{\boldsymbol{X}^{\prime} \boldsymbol{X}}^{-1}$ as the generalized inverse of matrix $\boldsymbol{X}^{\prime} \boldsymbol{X}$, and redefine $\boldsymbol{X}:=$

$574 \sum_{\boldsymbol{X}^{\prime} \boldsymbol{X}}^{-1 / 2} \boldsymbol{X}$. For input matrix $\boldsymbol{Y}$, we denote $\boldsymbol{\Sigma}_{\boldsymbol{Y} \boldsymbol{Y}^{\prime}}^{-1}$ as the generalized inverse of matrix $\boldsymbol{Y} \boldsymbol{Y}^{\prime}$, and redefine $575 \quad \boldsymbol{Y}:=\boldsymbol{Y} \Sigma_{\boldsymbol{Y} \boldsymbol{Y}}^{-1 / 2}$. The standardization of $\boldsymbol{Z}_{r}$ and $\boldsymbol{Z}_{l}$ is the same as above. 


\section{Algorithm 2. Calculating CCVs using SVD}

578

579

580

581

582

583

584

\section{5}

586

587

Take subproblem from the Equation (4) as an example, the goal of this module is to find projection matrix $\boldsymbol{U} \in \mathbb{R}^{K \times E}$ and $\boldsymbol{S} \in \mathbb{R}^{L \times E}$ such that the correlations between two indices $\boldsymbol{X} \boldsymbol{U}$ and $\boldsymbol{Z}_{l} \boldsymbol{S}$ are maximized.

$$
\underset{U, S}{\operatorname{argmax}} \operatorname{tr}\left(U^{\prime} X^{\prime} Z_{l} S\right) \text { subject to } U^{\prime} U=I, S^{\prime} S=I .
$$

We define $\Sigma_{\boldsymbol{X}^{\prime} \boldsymbol{Z}_{l}}:=\boldsymbol{X}^{\prime} \boldsymbol{Z}_{l}$. Let $\boldsymbol{U} \in \mathbb{R}^{K \times D}$ and $\boldsymbol{S} \in \mathbb{R}^{K \times D}$ be the matrices of the first $E$ left- and right singular vectors of $\Sigma_{\boldsymbol{X}^{\prime}} \boldsymbol{Z}_{l}$. Then the optimum in Equation (A1) is solved.

\section{Algorithm 3. Updating transition matrix with orthogonality constraints}

Take subproblem from the Equation (5) as an example, the goal of this module is to optimize $\boldsymbol{Z}_{l}$.

$$
\left(\boldsymbol{Z}_{l}\right):=\underset{\boldsymbol{Z}_{l}}{\operatorname{argmax}} \operatorname{tr}\left(\boldsymbol{U}^{\prime} \boldsymbol{X}^{\prime} \boldsymbol{Z}_{l} \boldsymbol{S}\right), \text { subject to } \boldsymbol{S}^{\prime} \boldsymbol{Z}_{l}^{\prime} \boldsymbol{Z}_{l} \boldsymbol{S}=\boldsymbol{I}
$$

Equation (A2) is maximized when $\boldsymbol{Z}_{l} \boldsymbol{S}=\boldsymbol{X} \boldsymbol{U}$. Therefore, we can update $\boldsymbol{Z}_{l}$ as

$$
\left(Z_{l}\right):=X \boldsymbol{U} \boldsymbol{S}^{\prime} \boldsymbol{\Sigma}_{S S^{\prime}}^{-1}
$$

where $\boldsymbol{\Sigma}_{S S^{\prime}}^{-1}$ denotes the generalized inverse of matrix $\boldsymbol{S} \boldsymbol{S}^{\prime}$.

\section{Parameter optimization}

There are two key hyperparameters when running bindSC for integration. The first one is the dimensionality $E$ in the latent space and the second one is the couple coefficient $\alpha$. Similar with previous integration methods, the number of dimension $E$ is very important on cell type classification. We provide heuristics to guide the selection of $E$ based on integration metrics defined below, though sometimes helpful, are not substitute for biological insights. As a general suggestion, we recommend starting with a value of $E$ the same with the minimal number of 
599 principle components (PCs) used on single modality clustering. The selection of couple coefficient

$600 \alpha$ depends on whether initialized $\boldsymbol{Z}^{(\mathbf{0})}$ can represent the gene score of $\boldsymbol{Y}$. We devise two metrics to

601 aid in selecting $\alpha$, which measure integration performance on accuracy (no mixing of cell type)

602 and alignment (mixing of datasets) as defined below.

\section{1) Silhouette score}

605 To measure integration accuracy, we use the Silhouette score. Cluster for each cell is defined 606 using the cell type labels assigned from single dataset clustering. The Silhouette score assesses the 607 separation of cell types, where a high score suggests that cells of the same cell type are close 608 together and far from cells of a different type. The Silhouette score $s(i)$ for each cell is calculated 609 as following. Let $a(i)$ be the average distance of cell $i$ to all other cells within $i$ 's cluster and $b(i)$

610 the average distance of $i$ to all cells in the nearest cluster, to which cell $i$ does not belong. Cell-cell

611 distance is computed in the L2-normalized co-embeddings (Equation 12). $s(i)$ can be computed 612 as:

$$
s(i)= \begin{cases}1-\frac{a(i)}{b(i)} & \text { if } a(i)<b(i) \\ 0 & \text { if } a(i)=b(i) \\ \frac{b(i)}{a(i)}-1 & \text { if } a(i)>b(i)\end{cases}
$$

614 Notably, given accurate correspondence between two modalities unknown, calculating s $(i)$ for cell

$615 i$ in above equation only includes cells from the same dataset. We average values across all cells 616 to obtain an overall silhouette score for integration task.

\section{2) Alignment mixing score}


619 To measure integration mixing level, we use an alignment mixing score similar to those of previous

620 studies ${ }^{50}$. We first build a 20-nearest neighbor graph for each cell from L2-normalized co-

621 embeddings (Equation 12). For cell $i$, assuming proportions of cells from two modalities are $p_{1 i}$

622 and $p_{2 i}$, respectively, the alignment mixing score is calculated as

$$
H(i)=-p_{1 i} \log _{2} p_{1 i}-p_{2 i} \log _{2} p_{2 i}
$$

624 This corresponds to a mixing metric per cell, and we average values across all cells to obtain an

625 overall mixing metric.

626

627 We run bindSC by ranging $a$ from 0 to 1 (with step size 0.1 ). Silhouette score and alignment

628 mixing score is calculated for each scenario. We select appropriate $a$ that generally has best

629 performance in Silhouette score and alignment mixing score. Parameter values used in this study

630 can be seen in Supplementary Table $\mathbf{S 1 .}$

631

\section{Performance and benchmarking}

633 In our evaluation, in addition to Silhouette score and alignment mixing score, we also consider

634 anchoring distance for evaluation datasets from multi-omics technologies, in which each cell has

635 paired profiles. For cell $i$ from the first data, we calculate its distance (Euclidean distance) with all

636 cells in the second data as $\boldsymbol{D}_{i}$, and its distance with cell $i$ in the second data as $d_{i}$. The anchoring

637 distance for cell $i$ is calculated as $2 d_{i} / \max \left(\boldsymbol{D}_{i}\right)$. We then average anchoring distance across all

638 cells to obtain an overall anchor distance metric. The anchoring distance of cell $i$ is 0 when it is

639 anchored correctly. The overall anchoring distance is 1 if we randomly layout cells on co640 embeddings. 


\section{Simulation dataset}

643 We generated simulation dataset to evaluate method performance in integrating two modalities 644 assuming accurate feature matching unknown. We used Splatter tool ${ }^{26}$ to simulate dataset $\boldsymbol{X}$ with 6451,000 genes and cells with different sizes (from 1,000 to 10,000). The whole population is 646 consisted of three batches (cell types). To mimic the feature unmatching case, we first generated 647 dataset $\boldsymbol{X}_{1}$ by randomly permutating genes of $\boldsymbol{X}$ under specified misalignment rates (MR). MR 648 ranges from 0 to 1 with step size being 0.05 in this study. $\boldsymbol{X}_{1}$ is the same as $\boldsymbol{X}$ if $\mathrm{MR}=0$. Half of 649 genes are matched between $\boldsymbol{X}_{1}$ and $\boldsymbol{X}$ if MR $=0.5$. No genes are matched between $\boldsymbol{X}_{1}$ and $\boldsymbol{X}$ if MR $650=1$. Then we generated matrix $\boldsymbol{Z}$ by adding $\boldsymbol{X}_{1}$ with white noise at certain level (i.e., Signal-Noise651 Ratio; SNR). SNR is set to be three levels (0, 0.25 and 0.5$)$.

653 For method comparison, previous methods including traditional CCA, Seurat ${ }^{17}$, Liger ${ }^{19}$, and 654 Harmony tools ${ }^{18}$ take $\boldsymbol{X}$ and $\boldsymbol{Z}$ as input assuming that cell correspondence between them is 655 unknown. bindSC takes two parts as input: 1) $\boldsymbol{X}$ and $\boldsymbol{Z}$ with cell correspondence unknown; 2) $\boldsymbol{X}$ 656 and $\boldsymbol{Z}$ with feature-level matching unknown (Supplementary Fig. 2).

\section{Preparation of dexamethasone (DEX) treated A549 cell dataset}

659 To investigate the ability of bindSC in integrating scRNA-seq and scATAC-seq profiles, we 660 explored the DEX-treated A549 dataset generated from sci-CAR technology, which uses 661 combinatorial indexing-based assay to jointly profile chromatin accessibility and mRNA on same 662 cell $^{6}$. In the A549 dataset, DEX is a synthetic corticosteroid which activates glucocorticoid 663 receptor (GR), binds to thousands of locations, and alternates the expression of hundreds of genes 664 51. The human lung adenocarcinoma derived A549 cells after 0, 1, or 3 hours of 100nM DEX 
665 treatment are assayed. The sci-RNA-seq dataset was from GSE117089

666 (https://www.ncbi.nlm.nih.gov/geo/query/acc.cgi?acc=GSE117089) and sci-ATAC-seq data was

667 from GSM3271041 (https://www.ncbi.nlm.nih.gov/geo/query/acc.cgi?acc=GSM3271041 ). The

668 original A549 data includes sci-RNA-seq profiles for 6,150 cells and sci-ATAC-seq profiles for

669 6,260 cells. There are 1,429 cells co-assayed. Following Cao et al., pre-processing pipeline

$670 \quad$ (https://github.com/KChen-

671 lab/bindSC/blob/master/vignettes/A549/A549 preprocess.ATAC.Rmd), we binarized peak count

672 matrix for cells from both ATAC-seq only and co-assay. Loci present in less than 5 cells and cells

673 with less than 300 accessible loci were removed. Peaks within $1 \mathrm{~kb}$ were merged and reads in

674 merged peaks were aggregated to generate a merged peak matrix, leading to 3,628 cells with

67532,791 loci. Each locus' accessibility in each cell was calculated by dividing the cell's raw read

676 count by cell specific size factor using estimateSizeFactors function in Monocle $2^{52}$. For RNA-

677 seq data, cells with expression counts less than 500 and more than 9100 were removed. The gene

678 expression in each cell was also calculated by dividing the cell's raw read count by cell specific

679 size factor, followed by $\log 2$ normalization. Genes with no variation in expression across cells

680 were further removed. The gene activity matrix was collapsed from the peak matrix by summing

681 all counts with the gene body plus $2 \mathrm{~kb}$ upstream using CreateGeneActivityMatrix function in

682 Seurat $3{ }^{17}$. We then picked top 10,000 variable genes in both sci-RNA-seq data and gene activity

683 data and used the overlapped 4,759 genes between them for integration. Finally, the sci-RNA-seq

684 matrix was composed of 6,005 cells with 4,759 genes, the gene activity matrix was composed of

6853,628 cells with 4,758 genes, and the sci-ATAC-seq matrix was composed of 3,628 cells with

68624,953 loci. There were 1,429 cells co-assayed.

687 


\section{Preparation of the mouse skin cell data}

689 We examined the performance of bindSC in integrating the scRNA-seq and scATAC-seq data

690 derived from mouse skin tissue. This dataset was generated using SHARE-seq (3) which included

69134,774 cells that have joint profiles of RNA and ATAC profiles. The RNA data was downloaded

692 from https://www.ncbi.nlm.nih.gov/geo/query/acc.cgi?acc=GSM4156608. The ATAC data was

693 downloaded from https://www.ncbi.nlm.nih.gov/geo/query/acc.cgi?acc=GSM4156597. The final

694 ATAC-seq matrix includes 25,594 cells on 74,161 peaks after quality control (including removing

695 cells with less than 350 genes expressed; peaks that exist in less than 500 cells). In addition, 4,894

696 genes were identified that were highly variable in both gene expression and gene activity profiles.

697 For this evaluation, we only focused on the third metric (e.g., anchoring distance) that represents

698 the chance for the two instances of a co-assayed cell to appear in the co-embeddings.

700 Preparation of the mouse retina 10x Genomics Multiome ATAC+RNA data

701 One mouse retina was dissociated by papain-based enzymatic digestion as described previously ${ }^{53}$

702 with slight modifications. Briefly, $45 \mathrm{U}$ of activated papain solution (with $1.2 \mathrm{mg}$ L-cysteine

703 (Sigma) and 1200U of DNase I (Affymetrix) in 5ml of HBSS buffer) was added to the tissue and

704 incubated at $37{ }^{\circ} \mathrm{C}$ for 20 minutes to release live cells. Post-incubation, papain solution was

705 replaced and deactivated with ovomucoid solution (15 mg ovomucoid (Worthington biochemical)

706 and $15 \mathrm{mg}$ BSA (Thermo Fisher Scientific) in $10 \mathrm{ml}$ of MEM (Thermo Fisher Scientific)). The

707 remaining tissue clumps were further triturated in the ovomucoid solution and filtered through a

708 20nm nylon mesh. After centrifugation at 300g 10min at 4C, the singe cells were resuspended PBS

709 with $0.04 \%$ BSA and checked for viability and cell count. About 1 million cells were pelleted and

710 resuspend in chilled lysis buffer (10x Genomics), incubate for 2 minutes on ice while monitored 
711 under microscope. $1 \mathrm{ml}$ of chilled wash buffer (10x Genomics) was added and sample was spun

712 down at 500g 5min at 4C and washed before resuspended in Diluted Nuclei Buffer (10x Genomics).

713 Nuclei concentration was determined using countess and proceed with transposition according to

714 manufacturer's recommendation (10x Genomics). After incubation for one hour at 37C, the

715 transposed nuclei were combined with barcoded gel beads, RT mix and partition oil on Chromium

716 to generate gel beads in Emulsion (GEMs). Single cell ATACseq library and 3'RNAseq library

717 were subsequently generated following recommended protocol from 10x Genomics. Libraries

718 were quantified and loaded on Novaseq 6000 and run with the following parameter: 151, 8, 8,

719 151bp. Data was analyzed using bcl2fastq (to generate fastq files) and cellranger pipeline (10x

720 Genomics).

721

722 Preparation of the mouse frontal cortex cell data

723 We investigate bindSC ability in integrating spatially resolved transcriptomic (ST) with

724 dissociated scRNA-seq. For the ST dataset, we used sagittal mouse brain slices generated from the

725 Visium v1 chemistry. The dataset was downloaded from https://support.10xgenomics.com/spatial-

726 gene-expression/datasets. The pre-processing workflow was guided by the Seurat3

727 (https://satijalab.org/seurat/v3.2/spatial_vignette.html). Briefly, cells were subset from anterior

728 region, followed by sctransform ${ }^{54}$. We then proceed to run dimensionality reduction and clustering

729 using standard workflow as did for scRNA-seq. Cluster ID 1,2,3,5,6,7 was extracted, followed by

730 segment based on exact position (Details in Subset out anatomical regions part in Seurat3

731 tutorial), leading to 1,072 cortical cells left for the ST data. One cortical scRNA-seq data composed

732 of $\sim 14,000$ adult mouse cortical cell taxonomy from the Allen Institute was collected

733 (https://www.dropbox.com/s/cuowvm4vrf65pvq/allen_cortex.rds?dl=1). This dataset was 
734 generated using the SMART-Seq2 protocol ${ }^{55}$. The sctransform normalization was performed

735 based on 3,000 variable genes. We used the cell type annotation provided by published meta data

736 available. There was a total of 14,294 cortical cells with 34,617 genes for the scRNA-seq data.

737 Integration of scRNA-seq and ST is based on 2,316 variable genes overlapped between two

738 datasets.

740 To predict locations of each cell type from scRNA-seq in the histological images, we built a 741 support vector machine (SVM) that trained on cell profiles from scRNA-seq data. In the training 742 model, features were identified as cell coordinates in co-embeddings and labels were 743 corresponding cell types. The trained SVM was applied to ST data and output predicted probability 744 of each cell type at each spot. The SpatialFeaturePlot function in Seurat3 was used to overlay 745 predicted probabilities for each cell type on top of tissue histology.

\section{Preparation of human bone marrow cell dataset}

748 We examined the performance of bindSC in integrating the single-cell RNA and protein data

749 derived from human bone marrow tissue. This dataset was generated using the CITE-seq

750 technology ${ }^{40}$, which included 30,672 cells that have joint profiles of RNA and a panel of 25

751 antibodies. The dataset was fom

752 https://satijalab.org/seurat/v4.0/weighted_nearest_neighbor_analysis.html. We extracted the 25

753 protein-homologous gene expression profile from the RNA data and kept cells that have total

754 expression count $>2$. The final protein matrix includes 28,609 cells with 25 protein abundance

755 levels. The gene expression matrix includes 28,609 cells with 3,000 genes. The protein-

756 homologous RNA matrix includes 28,609 cells with the RNA levels of the 25 genes homologous 
757 to the 25 proteins. To measure anchoring accuracy for each cell type, we used the third metric,

758 anchoring distance, which measures the distance of protein and gene expression for each cell in

759 co-embeddings.

760

761 Motif-based Transcription Factors (TFs) activity estimation

762 To estimate transcription factor activity from scATAC-seq data, we used default settings in

763 chromVAR ${ }^{56}$ package. This approach quantifies accessibility variation across single cells by

764 aggregating accessible regions containing a specific TF motif. It calculated motif-based TF activity

765 by comparing the observed accessibility of all the peaks containing a TF motif to a background set

766 of peaks normalizing against known technical confounders.

767

768 Acknowledgements

769 This project has been made possible in part by the Human Cell Atlas Seed Network Grant

770 CZF2019-02425 to RC and KC, CZF2019-002432 to KC from the Chan Zuckerberg Initiative

771 DAF, an advised fund of Silicon Valley Community Foundation, grant R01EY022356 and

772 R01EY018571 to RC from National Eye Institute, grant RP180248 to KC from Cancer Prevention

$773 \&$ Research Institute of Texas, grant U01CA247760 to KC and the Cancer Center Support Grant

774 P30 CA016672 to PP from National Cancer Institute. This project was also partially supported by

775 the Single Cell Genomics Core at Baylor College of Medicine funded by NIH shared instrument

776 grants (S10OD023469, S10OD025240) and P30EY002520. The authors would like to thank

777 Qingnan Liang, Yuanxin Wang, Linghua Wang, Tapsi Kumar, Runmin Wei, Nicholas Navin, John

778 Weinstein and Hussein Abbas for their comments.

779 


\section{Author contributions}

781 K.C. conceptualized and supervised the project. J.D. designed the bindSC tool, implemented the

782 software and performed analysis. R.C., Y. L., X. C., S.K., J.C., contributed to mouse retina 10x

783 Genomics ATAC+RNA data generation, curation. V.M. contributed to data interpretation. J.D.,

784 S.L. and K.C. drafted the manuscript. All authors reviewed, edited, and approved the manuscript.

785

\section{Competing interests}

787 The authors declare no competing interests. 


\section{Reference}

7891 Macosko, E. Z. et al. Highly parallel genome-wide expression profiling of individual cells

$790 \quad$ using nanoliter droplets. Cell 161, 1202-1214 (2015).

7912 Spitzer, M. H. \& Nolan, G. P. Mass cytometry: single cells, many features. Cell 165, 780-

792

7933 791 (2016). Luo, C. et al. Single-cell methylomes identify neuronal subtypes and regulatory elements in mammalian cortex. Science 357, 600-604 (2017).

7965 Mulqueen, R. M. et al. Highly scalable generation of DNA methylation profiles in single cells. Nature biotechnology 36, 428-431 (2018).

7986 Cao, J. et al. Joint profiling of chromatin accessibility and gene expression in thousands of single cells. Science 361, 1380-1385 (2018).

8007 Cusanovich, D. A. et al. A single-cell atlas of in vivo mammalian chromatin accessibility. Cell 174, 1309-1324. e1318 (2018). the human adult brain. Nature biotechnology 36, 70-80 (2018). Moffitt, J. R. et al. Molecular, spatial, and functional single-cell profiling of the hypothalamic preoptic region. Science 362 (2018). states. Science 361 (2018). genome and transcriptome sequencing of the same cell. Nature biotechnology 33, 285-289 (2015).

transcriptomes. Nature methods 12, 519-522 (2015).

13 Argelaguet, R. et al. Multi-omics profiling of mouse gastrulation at single-cell resolution. Nature 576, 487-491 (2019).

14 Ma, S. et al. Chromatin Potential Identified by Shared Single-Cell Profiling of RNA and Chromatin. Cell (2020).

15 Zhu, C., Preissl, S. \& Ren, B. Single-cell multimodal omics: the power of many. Nature methods 17, 11-14 (2020).

16 Wang, C. et al. Integrative analyses of single-cell transcriptome and regulome using MAESTRO. Genome biology 21, 1-28 (2020).

17 Stuart, T. et al. Comprehensive integration of single-cell data. Cell 177, 1888-1902. e1821 (2019).

18 Korsunsky, I. et al. Fast, sensitive and accurate integration of single-cell data with Harmony. Nature methods, 1-8 (2019).

19 Rosenberg, A. B. et al. Single-cell profiling of the developing mouse brain and spinal cord with split-pool barcoding. Science 360, 176-182 (2018).

20 Singh, R. et al. Unsupervised manifold alignment for single-cell multi-omics data. BioRxiv (2020).

21 Cao, K., Bai, X., Hong, Y. \& Wan, L. Unsupervised Topological Alignment for SingleCell Multi-Omics Integration. bioRxiv (2020). 
83122 Welch, J. D., Hartemink, A. J. \& Prins, J. F. MATCHER: manifold alignment reveals

862

863

864

865

866

867

868

869

870

871

872

873

874

875

876 correspondence between single cell transcriptome and epigenome dynamics. Genome biology 18, 1-19 (2017).

23 Pliner, H. A. et al. Cicero predicts cis-regulatory DNA interactions from single-cell chromatin accessibility data. Molecular cell 71, 858-871. e858 (2018).

24 Lieberman-Aiden, E. et al. Comprehensive mapping of long-range interactions reveals folding principles of the human genome. science 326, 289-293 (2009).

25 Lara-Astiaso, D. et al. Chromatin state dynamics during blood formation. science 345, 943949 (2014).

26 Zappia, L., Phipson, B. \& Oshlack, A. Splatter: simulation of single-cell RNA sequencing data. Genome biology 18, 1-15 (2017).

27 Jin, S., Zhang, L. \& Nie, Q. scAI: an unsupervised approach for the integrative analysis of parallel single-cell transcriptomic and epigenomic profiles. Genome biology 21, 1-19 (2020).

28 Consortium, E. P. An integrated encyclopedia of DNA elements in the human genome. Nature 489, 57-74 (2012).

29 Liang, Q. et al. Single-nuclei RNA-seq on human retinal tissue provides improved transcriptome profiling. Nature communications 10, 1-12 (2019).

30 Masland, R. H. The neuronal organization of the retina. Neuron 76, 266-280 (2012).

31 Menon, M. et al. Single-cell transcriptomic atlas of the human retina identifies cell types associated with age-related macular degeneration. Nature communications 10, 1-9 (2019).

32 Clark, B. S. et al. Single-cell RNA-seq analysis of retinal development identifies NFI factors as regulating mitotic exit and late-born cell specification. Neuron 102, 1111-1126. e1115 (2019).

33 Shekhar, K. et al. Comprehensive classification of retinal bipolar neurons by single-cell transcriptomics. Cell 166, 1308-1323. e1330 (2016).

34 Brunet, I. et al. The transcription factor Engrailed-2 guides retinal axons. Nature 438, 9498 (2005).

35 Nishida, A. et al. Otx2 homeobox gene controls retinal photoreceptor cell fate and pineal gland development. Nature neuroscience 6, 1255-1263 (2003).

36 Marquardt, T. et al. Pax6 is required for the multipotent state of retinal progenitor cells. Cell 105, 43-55 (2001).

37 Ramanathan, M., Porter, D. F. \& Khavari, P. A. Methods to study RNA-protein interactions. Nature methods 16, 225-234 (2019).

38 Krishnaswamy, S. et al. Conditional density-based analysis of T cell signaling in singlecell data. Science 346 (2014).

39 Efremova, M. \& Teichmann, S. Computational methods for single-cell omics across modalities. Nature Methods 17, 14-17 (2020).

40 Stoeckius, M. et al. Simultaneous epitope and transcriptome measurement in single cells. Nature methods 14, 865 (2017).

41 Doyle, H. A. \& Mamula, M. J. Post-translational protein modifications in antigen recognition and autoimmunity. Trends in immunology 22, 443-449 (2001).

42 Granja, J. M. et al. ArchR: An integrative and scalable software package for single-cell chromatin accessibility analysis. bioRxiv (2020).

43 Rozenblatt-Rosen, O., Stubbington, M. J., Regev, A. \& Teichmann, S. A. The Human Cell Atlas: from vision to reality. Nature News 550, 451 (2017). 
87744 Consortium, H. The human body at cellular resolution: the NIH Human Biomolecular Atlas

$878 \quad$ Program. Nature 574, 187 (2019).

87945 Rozenblatt-Rosen, O. et al. The Human Tumor Atlas Network: charting tumor transitions

880 across space and time at single-cell resolution. Cell 181, 236-249 (2020).

88146 Sharma, A. et al. Onco-fetal Reprogramming of Endothelial Cells Drives

882 Immunosuppressive Macrophages in Hepatocellular Carcinoma. Cell 183, 377-394. e321

$883 \quad$ (2020).

88447 Warren, A. et al. Global computational alignment of tumor and cell line transcriptional

$885 \quad$ profiles. BioRxiv (2020).

88648 Hardoon, D. R., Szedmak, S. \& Shawe-Taylor, J. Canonical correlation analysis: An

887 overview with application to learning methods. Neural computation 16, 2639-2664 (2004).

88849 Traag, V. A., Waltman, L. \& van Eck, N. J. From Louvain to Leiden: guaranteeing well-

$89050 \quad$ Welch, J. D. et al. Single-cell multi-omic integration compares and contrasts features of brain cell identity. Cell 177, 1873-1887. e1817 (2019).

51 Vockley, C. M. et al. Direct GR binding sites potentiate clusters of TF binding across the human genome. Cell 166, 1269-1281. e1219 (2016). Qiu, X. et al. Reversed graph embedding resolves complex single-cell trajectories. Nature methods 14, 979 (2017).

53 Siegert, S. et al. Transcriptional code and disease map for adult retinal cell types. Nature neuroscience 15, 487-495 (2012).

54 Hafemeister, C. \& Satija, R. Normalization and variance stabilization of single-cell RNA-

55 Picelli, S. et al. Full-length RNA-seq from single cells using Smart-seq2. Nature protocols 9, 171-181 (2014).

57 Tasic, B. et al. Adult mouse cortical cell taxonomy revealed by single cell transcriptomics. Nature neuroscience 19, 335-346 (2016). 


\section{Figure Legends}

909 Fig. 1 Overview of bindSC. BindSC takes as input two data matrices produced by different modalities

910 from the same cell population (a). The modalities may include transcriptomes, epigenomes, spatial

911 transcriptomes and proteomes. Bi-order integration of two modalities $(\mathbf{X}$ and $\mathbf{Y})$ with unpaired cells and

912 unmatched features using Bi-CCA algorithm (b). In the data matrices, each row represents one gene/locus,

913 and each column represents one cell. The gene score matrix $\mathbf{Z}$ that links the first modality with the second

914 one is initialized by prior gene activity modeling (see Methods). Bi-CCA algorithm aims to update gene

915 score matrix $\mathbf{Z}$ iteratively by maximizing the correlation of between $\mathbf{X}$ and $\mathbf{Z}$ and between $\mathbf{Y}$ and $\mathbf{Z}$

916 simultaneously. Based on canonical correlation vectors (CCVs) in the derived latent space, K-nearest

917 neighbor (KNNs) clustering is performed to define cell types in both modalities (c). Within each cell-type

918 cluster, KNN clustering is further performed at a higher resolution to define pseudo-cells consisting of 10s

919 cells from both modalities. In silico multimodal profiles are constructed from cells assigned to the same

920 pseudo-cell (d). The color in each box indicates the relative level of each feature, with white corresponding

921 to missing values. The multiomics feature profiles enable us to 1) link genes to regulatory elements, 2) map

922 RNA expressions to spatial locations and 3) delineate cells by both RNA and protein signatures (e).

924 Fig. 2 Benchmarking bindSC performance on simulation datasets. Comparison of bindSC to CCA,

925 Seurat, LIGER, and Harmony based on Silhouette score and alignment mixing score (a). The dataset

926 contains 1,000 genes and 1,000 cells equally distributed in 3 cell types. Signal-to-noise ratio (SNR) was set

927 at 0.25 . X-axes denote the misalignment rates (MR) between features in the two datasets, which ranges

928 from 0 to 1 . The features between two datasets have perfect match if $\mathrm{MR}=0$ and are unrelated if $\mathrm{MR}=1$.

929 UMAP views of the co-embeddings generated by bindSC, CCA, Seurat, LIGER, and Harmony (b). From

930 top to bottom are results with $\mathrm{MR}=0.1,0.5$, and 0.9 , respectively. Each point denotes one cell that is

931 colored based on its true cell type label (red, green, or cyan). 


\section{Fig.3 Benchmarking bindSC performance on the DEX-treated A549 cell-line data. UMAP of cells}

934 from DEX-treated A549 cell-line data for bindSC, Seurat, LIGER and Harmony respectively, colored by

935 collection time (red: 0 hour, green: 1 hour and blue: 3 hour) on the top panel and by technologies (grey: sci-

936 RNA and brown: sci-ATAC) on the bottom panel (a). Comparison of the 4 methods based on Silhouette

937 score (top), alignment mixing score (middle) and anchoring distance (bottom) (b). Also included for

938 comparison are metrics from randomly selected cells. Accuracy of in silico multiomics profile in pseudo-

939 cells (c). The TF-gene correlation is quantified in each pseudo-cell (top panel) by calculating for each TF-

940 gene pair (1.8 million pairs total) a Spearman's rank-order correlation coefficient (SRCC) between the TF

941 activity level, inferred based on motif enrichment in the ATAC data, and the gene expression level in the

942 RNA data. The peak-gene correlation is quantified by calculating for each peak-gene pair a Spearman's

943 rank-order correlation coefficients (SRCC) between a normalized ATAC peak level and a gene expression

944 levels for the cis-peaks (middle panel, 7,833 pairs) and the trans-peaks (bottom panel, 118.7 million pairs),

945 respectively. X-axes are the SRCCs estimated from the co-assayed cells, which serve as the gold standard,

946 while Y-axes are the SRCCs estimated from the pseudo-cells generated by each method. The overall

947 concordance between X and Y are further quantified using a single SRCC shown on the up-left corner of

948 each subfigure. Cis is defined as gene bodies plus 2,000 bps upstream. Reconstructing the gene expression

949 and the TF activity level (Y-axes) of NR3C1 using bindSC pseudo-cells (d). X-axis is the averaged

950 treatment time of the cells in each pseudo-cell. A genome browser view showing putative regulatory

951 relations between an accessible distal site chr2:201770437-201770992 and the gene CFLAR (e). The 6

952 tracks at the top show ATAC peak levels and gene expression levels at six time points. The track in the

953 middle shows chromatin interactome from published $\mathrm{Hi}-\mathrm{C}$ data. The bottom track shows the NR3Cl binding

954 targets (ChIP-Seq) peaks published in an independent study ${ }^{28}$.

956 Fig. 4 Integrating single-cell RNA-seq and ATAC-seq on a mouse retinal cell atlas. UMAP views of

9579,383 mouse retina cells based on gene expression levels in the RNA-seq data (a), chromatin accessibility

958 peak profiles in the ATAC-seq data (b), gene-level collapsed chromatin accessibility profiles (c). The cells 
are colored by cell types annotated based on RNA expression levels (Supplementary Fig. 8). BindSC coembeddings for the cells in the RNA-seq data (d) and those in the ATAC-seq data (e). Anchoring distances

961 resulting from bindSC, Seurat, LIGER and Harmony (f). The median anchoring distance for each cell type

962 was highlighted as a bold horizonal bar in the middle of each box in each panel. The dotted line denotes the

963 anchoring distance based on random guesses. Zoomed out UMAP views for the BC cells in the co-

964 embeddings generated by bindSC (g), Seurat (h), LIGER (i), and Harmony (j). Integration results for all

965 the cell types can be seen in Supplementary Fig. 9. RGC: retinal ganglion cells; HC: horizontal cells; BC:

966 bipolar cells; AC: amacrine cells; MG: Müller glial cell.

Fig. 5 Integrating spatially resolved transcriptomic (ST) data with scRNA-seq data from mouse

frontal cortex. UMAPs of the gene expression levels for the 14,249 cells profiled by SMART-Seq2 and

971 publication ${ }^{57}$. Predicted locations of each cell type in the histological images (b). Color gradient

972 corresponds to the probability score of a cell being mapped to a particular spatial location. Comparison of

973 cell type frequencies estimated from the ST data (Y-axis) to those estimated from the scRNA-seq data (X-

974 axis) (c). Correlation coefficients (R) and $\mathrm{P}$ values are calculated based on Pearson's correlation analysis.

975 Each dot corresponds to a cell-type (labeled in different colors). The blue line and the grey shade represent

976 regression lines and 95\% confidence intervals from performing linear regressions.

Fig. 6 Integrating single-cell RNA with protein data produced by a CITE-seq assay. The UMAP of 30,672 human bone marrow cells based on 25 surface protein levels (a), 3,000 highly variable gene expression levels (b) and 25 protein-homologous gene expression levels (c). The cell type labels are from

981 the original study ${ }^{17}$. UMAP of the protein (d) and the RNA (e) expression data in the co-embedding 982 generated by bindSC. Comparison of anchoring distances generated by bindSC, Seurat, LIGER and 983 Harmony (f). The red dotted line denotes the anchoring distance from random guesses. Anchoring distances 984 for each cell type in the bindSC co-embedding (g). 
a

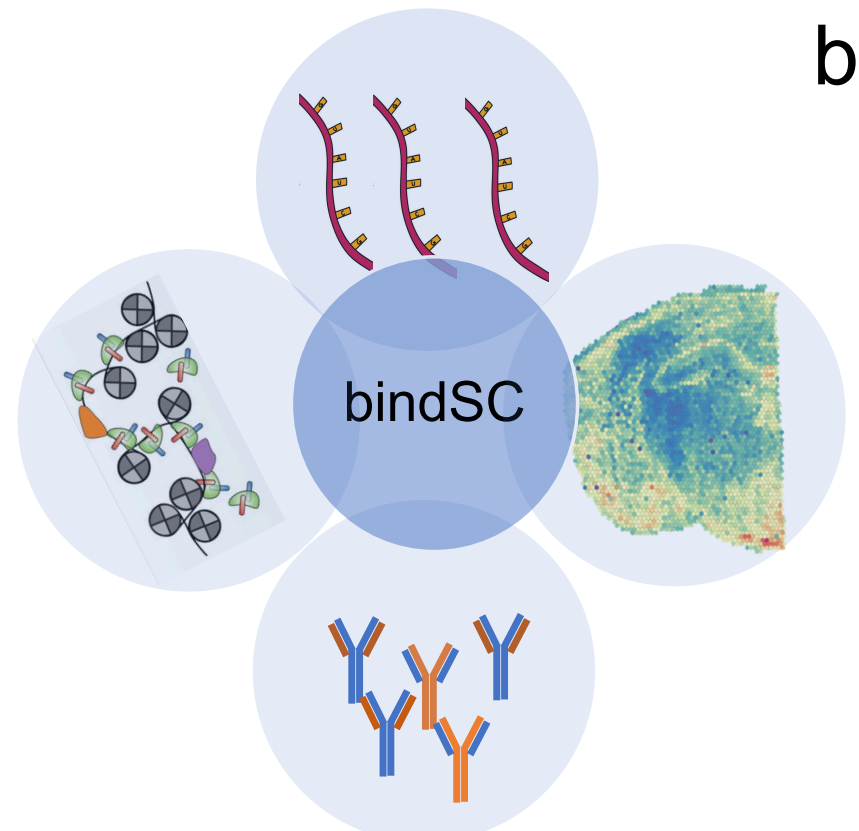

b Bi-CCA

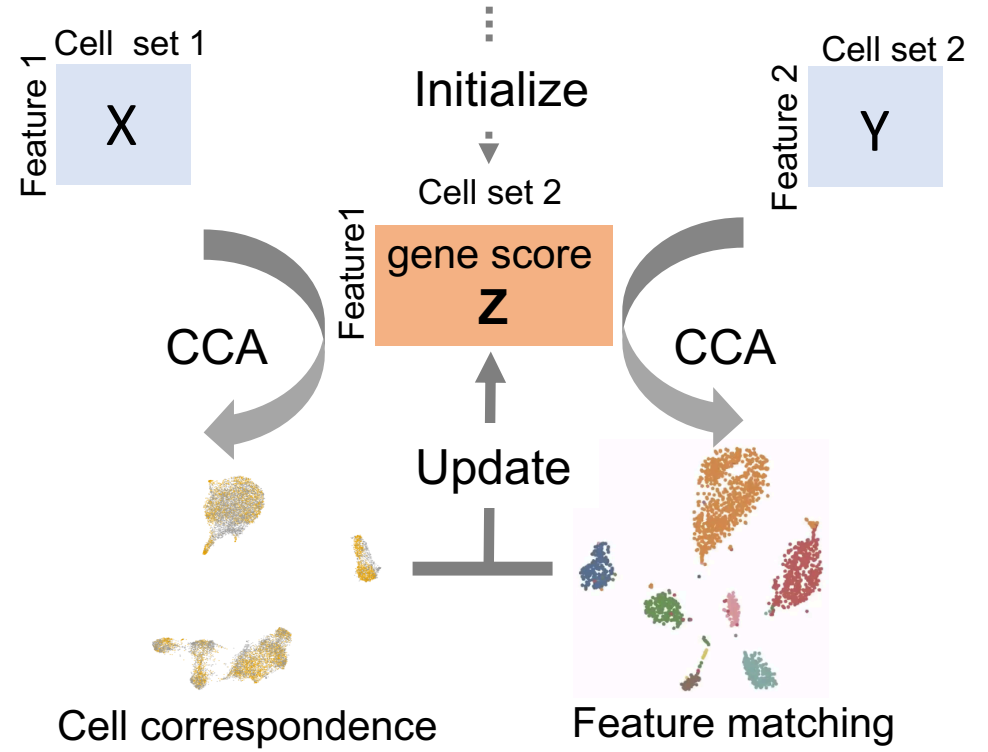

C

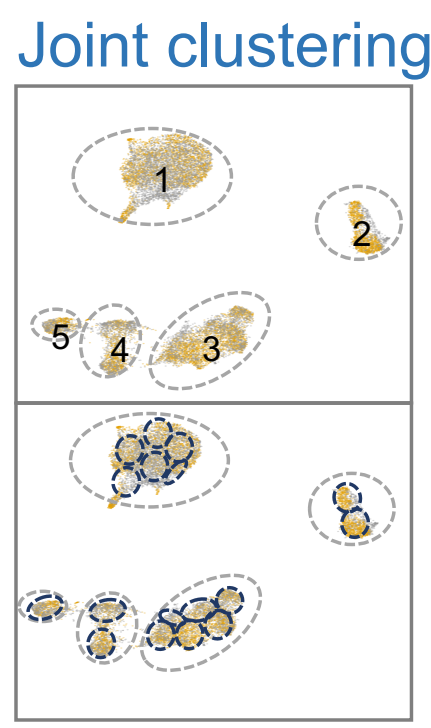

d Pseudo-cell profiles

\begin{tabular}{|c|c|c|c|c|c|c|c|}
\hline Cluster & Pseudo-cells & \multicolumn{3}{|c|}{ Feature1 } & \multicolumn{3}{|c|}{ Feature2 } \\
\hline & & $\mathrm{f}_{1}$ & $\mathrm{f}_{2}$ & $\mathrm{f}_{3}$ & $\mathrm{~g}_{1}$ & $\mathrm{~g}_{2}$ & $\mathrm{~g}_{3}$ \\
\hline 1 & $\mathrm{a}_{11}$ & & & & & & \\
\hline & $\mathrm{a}_{12}$ & & & & & & \\
\hline & $\mathrm{a}_{13}$ & & & & & & \\
\hline 2 & $\mathrm{a}_{21}$ & & & & & & \\
\hline & $\mathrm{a}_{22}$ & & & & & & \\
\hline & $\mathrm{a}_{23}$ & & & & & & \\
\hline 3 & $\mathrm{a}_{31}$ & & & & & & \\
\hline & $\mathrm{a}_{32}$ & & & & & & \\
\hline 4 & $\mathrm{a}_{41}$ & & & & & & \\
\hline & $\mathrm{a}_{51}$ & & & & & & \\
\hline 5 & $\mathrm{a}_{52}$ & & & & & & \\
\hline & $\mathrm{a}_{53}$ & & & & & & \\
\hline
\end{tabular}

e Multi-omics integration

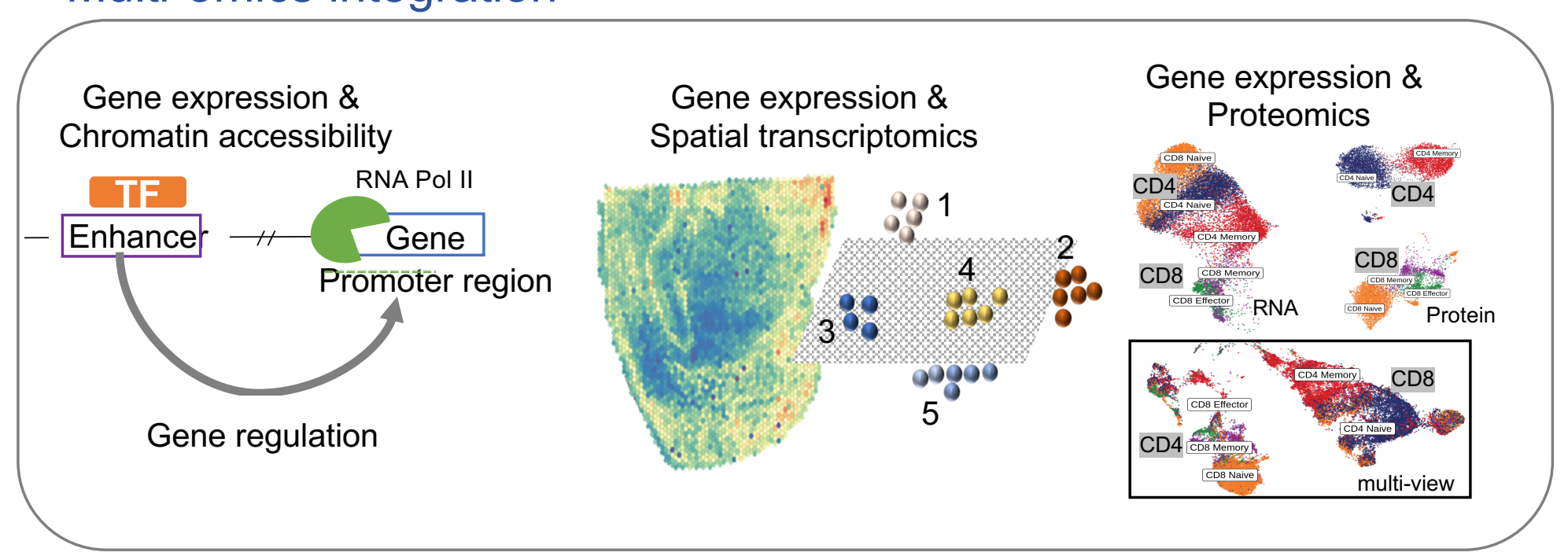

Fig. 1 Overview of bindSC. BindSC takes as input two data matrices produced by different modalities from the same cell population (a). The modalities may include transcriptomes, epigenomes, spatial transcriptomes and proteomes. Bi-order integration of two modalities ( $\mathbf{X}$ and $\mathbf{Y}$ ) with unpaired cells and unmatched features using $\mathrm{Bi}-$ CCA algorithm (b). In the data matrices, each row represents one gene/locus, and each column represents one cell. The gene score matrix $\mathbf{Z}$ that links the first modality with the second is initialized by prior gene activity modeling (see Methods). Bi-CCA algorithm aims to update gene score matrix $\mathbf{Z}$ iteratively by maximizing the correlation of between $\mathbf{X}$ and $\mathbf{Z}$ and between $\mathbf{Y}$ and $\mathbf{Z}$ simultaneously. Based on canonical correlation vectors (CCVs) in the derived latent space, $\mathrm{K}$-nearest neighbor (KNNs) clustering is performed to define cell types in both modalities (c). Within each cell-type cluster, KNN clustering is further performed at a higher resolution to define pseudo-cells consisting of 10 s cells from both modalities. In silico multimodal profiles are constructed from cells assigned to the same pseudo-cell (d). The color in each box indicates the relative level of each feature, with white corresponding to missing values. The multiomics feature profiles enable us to 1 ) link genes to regulatory elements, 2) map RNA expressions to spatial locations and 3) delineate cells by both RNA and protein signatures (e). 
a
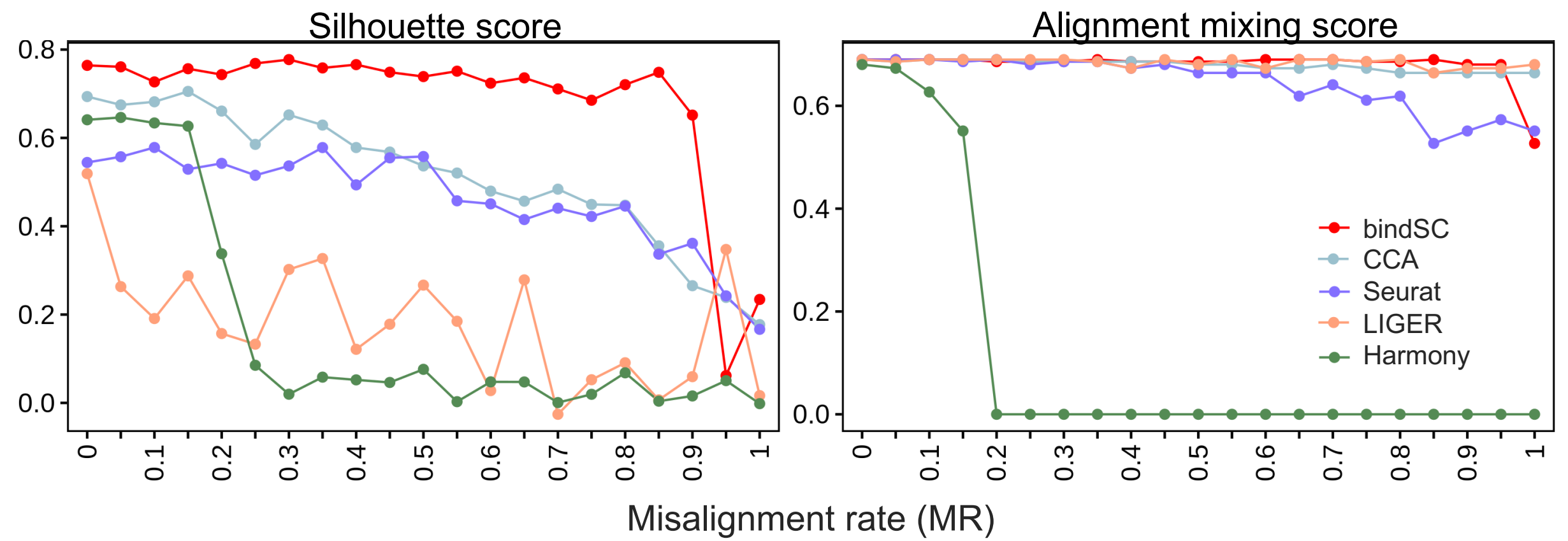

Misalignment rate (MR) b
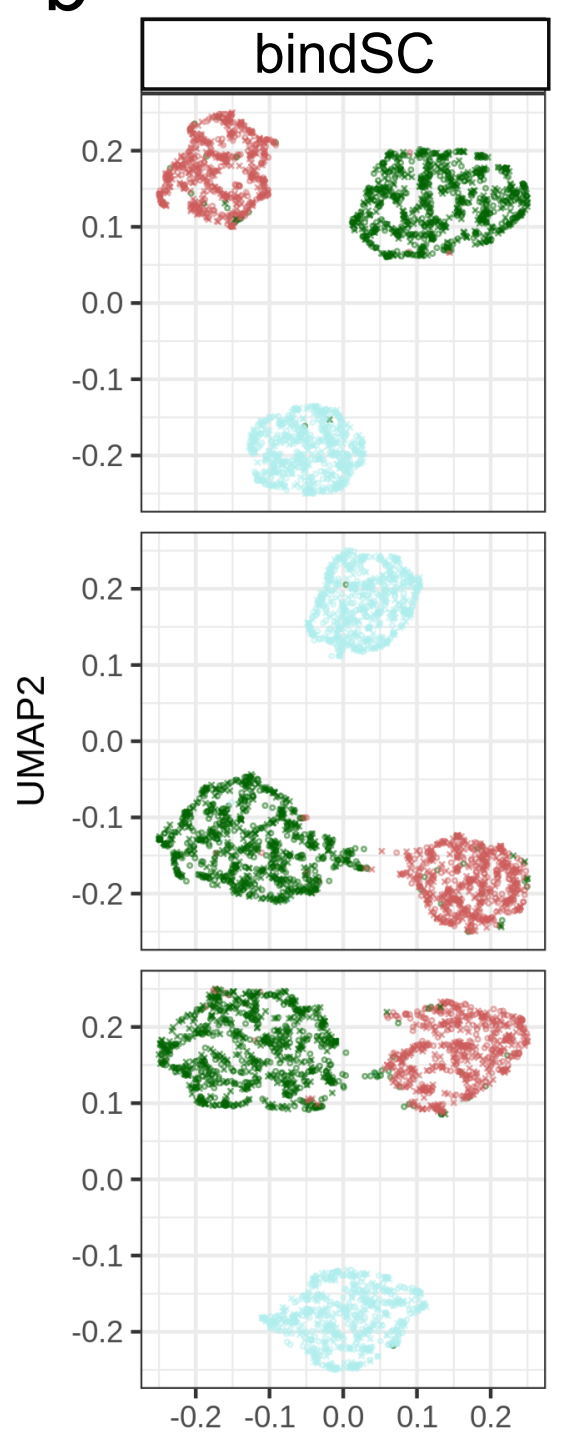
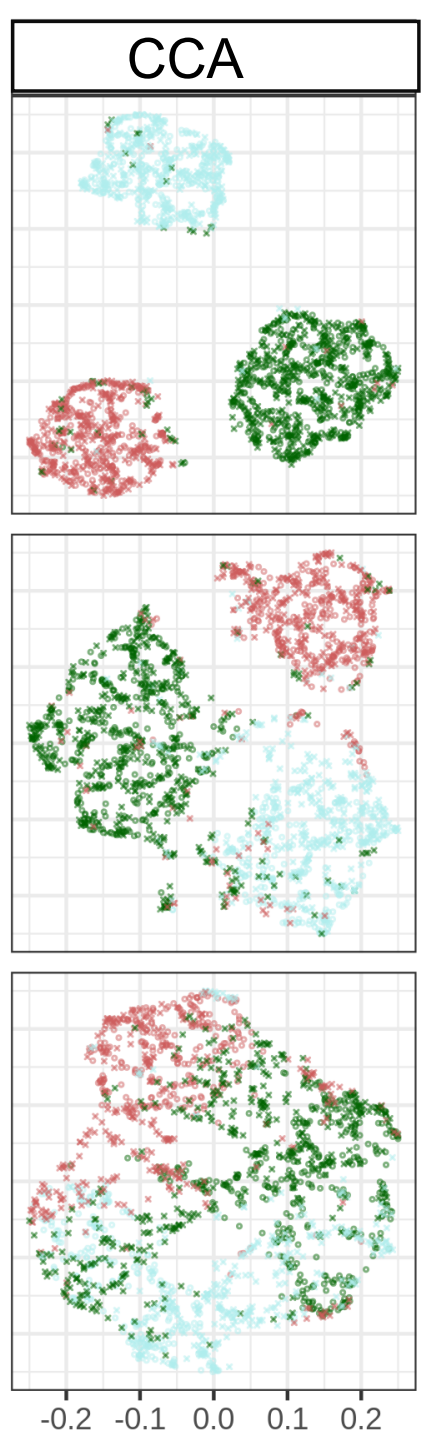
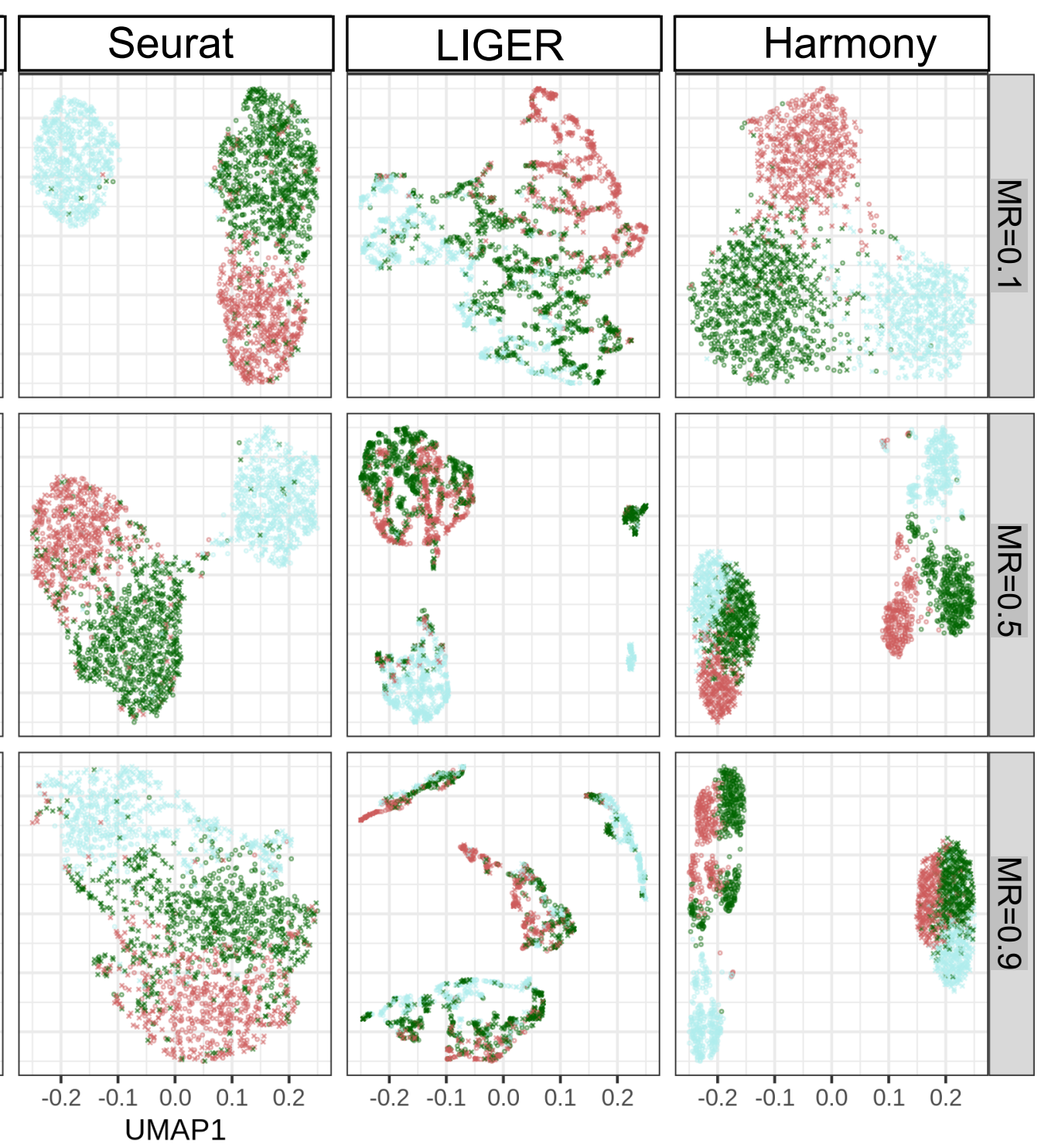

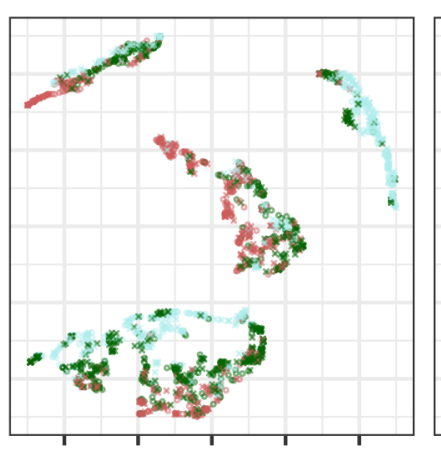

$\begin{array}{ccccc}-0.2 & -0.1 & 0.0 & 0.1 & 0.2\end{array}$

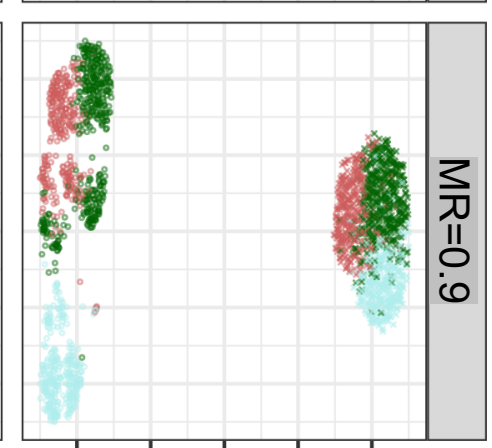

$\begin{array}{lllll}-0.2 & -0.1 & 0.0 & 0.1 & 0.2\end{array}$

Fig. 2 Benchmarking bindSC performance on simulation datasets. Comparison of bindSC to CCA, Seurat, LIGER, and Harmony based on Silhouette score and alignment mixing score (a). The dataset contains 1,000 genes and 1,000 cells equally distributed in 3 cell types. Signal-to-noise ratio (SNR) was set at 0.25 . X-axes denote the misalignment rates (MR) between features in the two datasets, which ranges from 0 to 1 . The features between two datasets have perfect match if $M R=0$ and are unrelated if $M R=1$. UMAP views of the co-embeddings generated by bindSC, CCA, Seurat, LIGER, and Harmony (b). From top to bottom are results with $M R=0.1,0.5$, and 0.9 , respectively. Each point denotes one cell that is colored based on its true cell type label (red, green, or cyan). 
Fig. 3

a
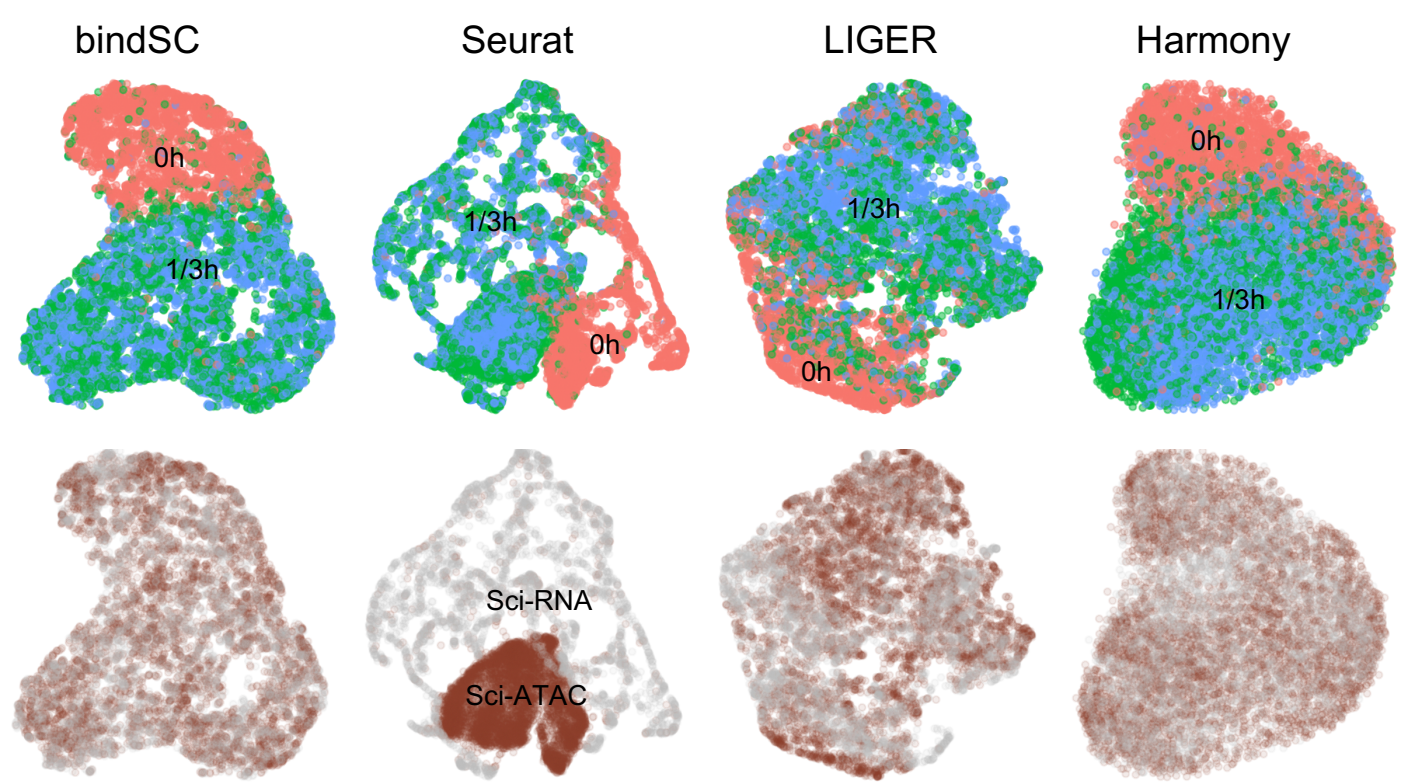

C

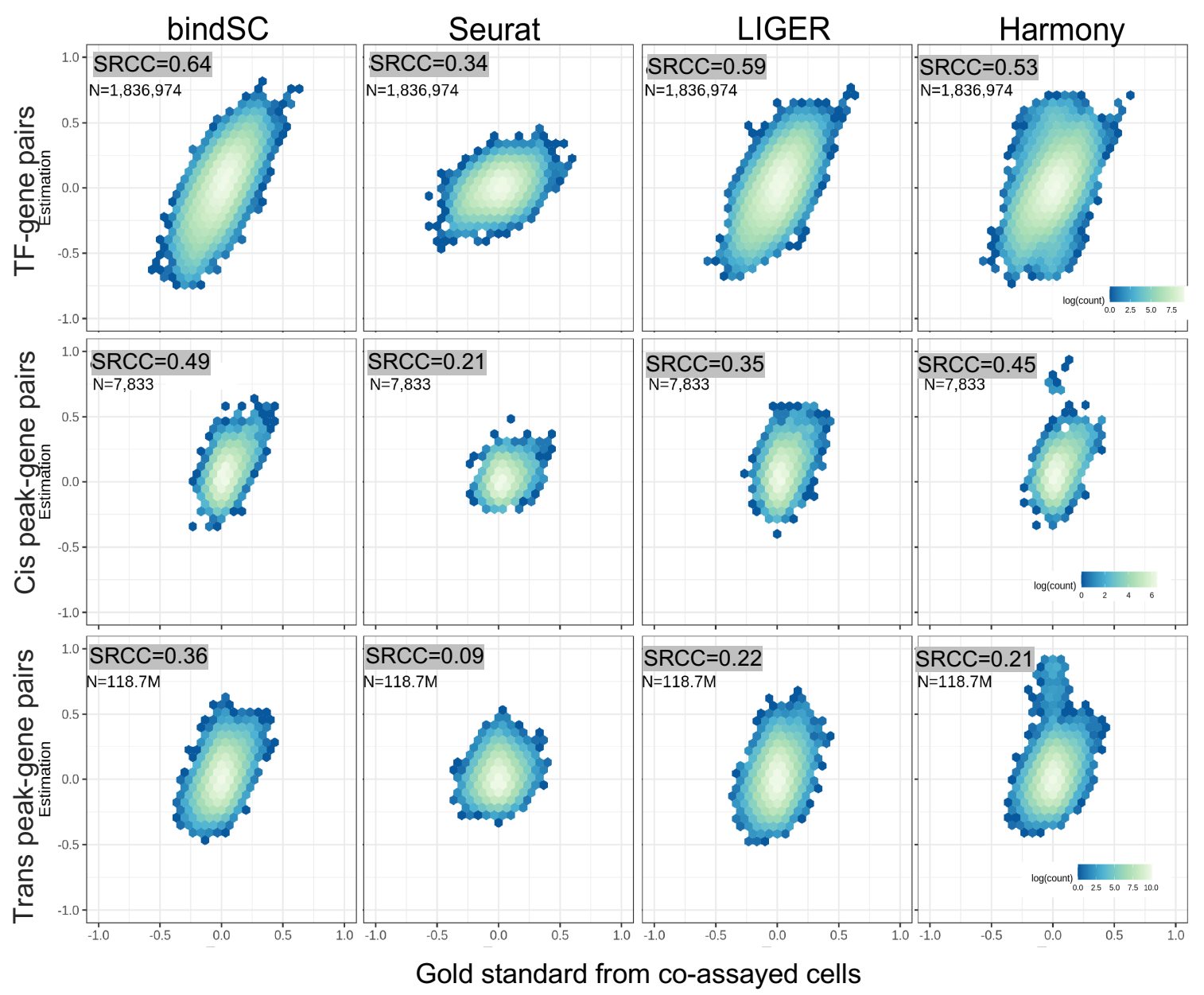

b
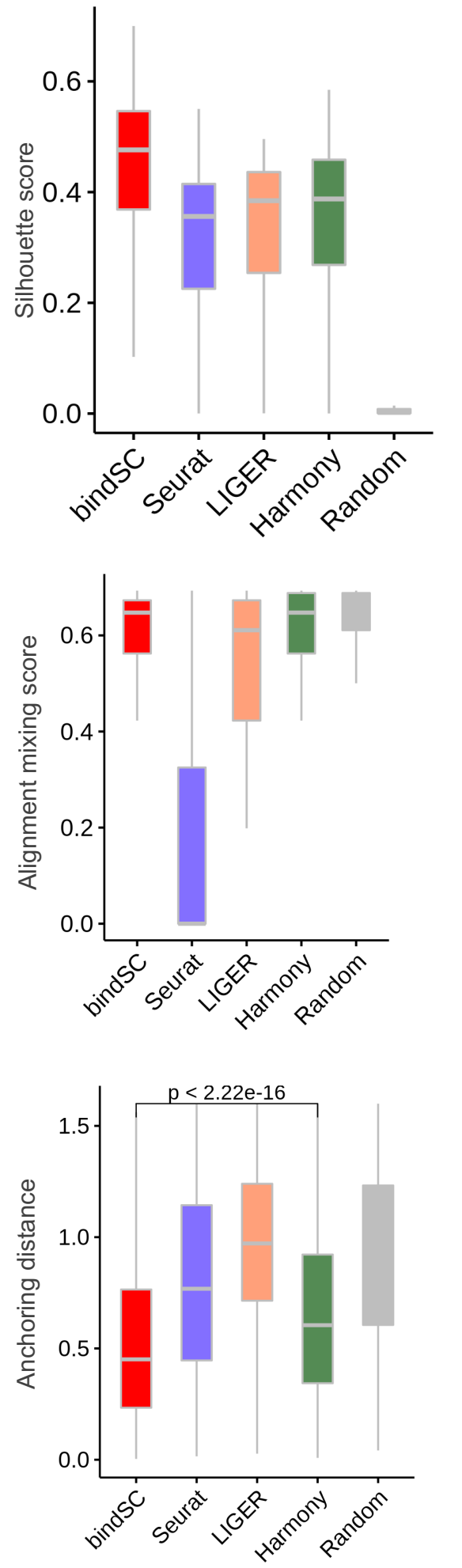

d

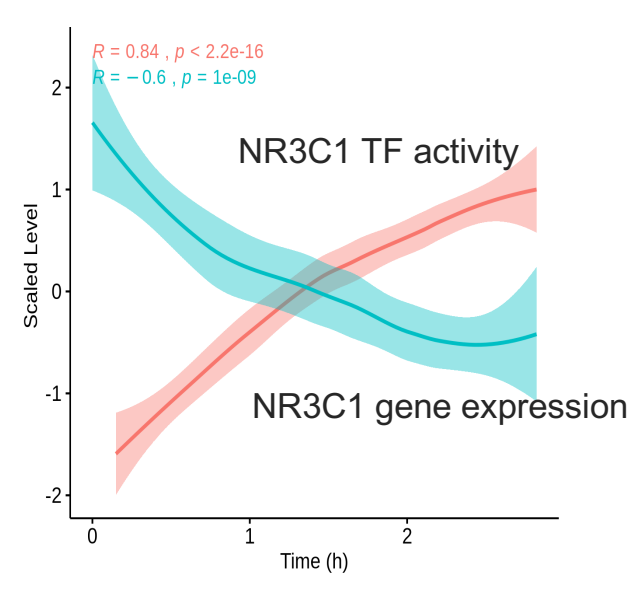

e

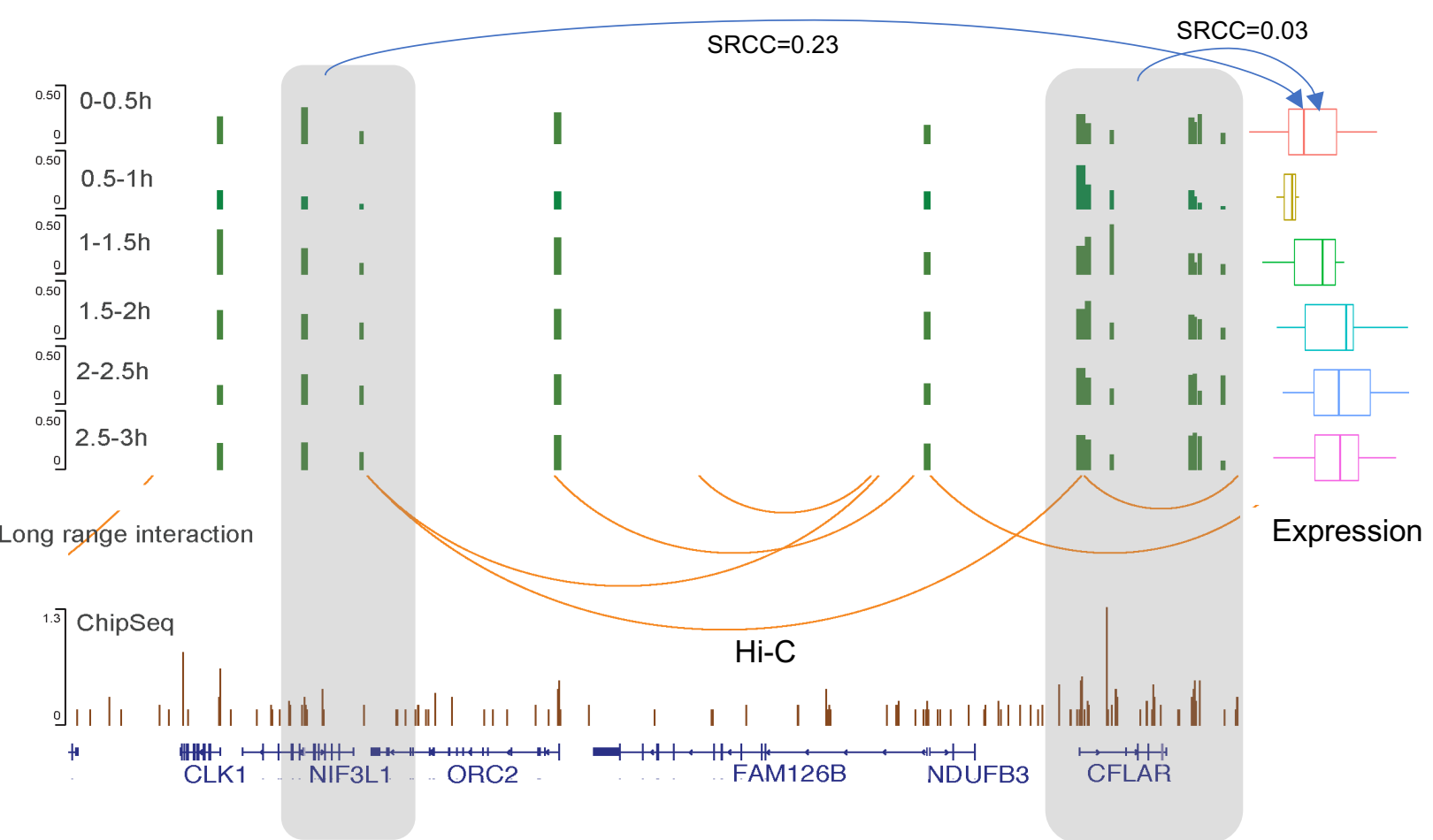


Fig.3 Benchmarking bindSC performance on the DEX-treated A549 cell-line data. UMAP of cells from DEXtreated A549 cell-line data for bindSC, Seurat, LIGER and Harmony respectively, colored by collection time (red: 0 hour, green: 1 hour and blue: 3 hour) on the top panel and by technologies (grey: sci-RNA and brown: sciATAC) on the bottom panel (a). Comparison of the 4 methods based on Silhouette score (top), alignment mixing score (middle) and anchoring distance (bottom) (b). Also included for comparison are metrics from randomly selected cells. Accuracy of in silico multiomics profile in pseudo-cells (c). The TF-gene correlation is quantified in each pseudo-cell (top panel) by calculating for each TF-gene pair (1.8 million pairs total) a Spearman's rankorder correlation coefficient (SRCC) between the TF activity level, inferred based on motif enrichment in the ATAC data, and the gene expression level in the RNA data. The peak-gene correlation is quantified by calculating for each peak-gene pair a Spearman's rank-order correlation coefficients (SRCC) between a normalized ATAC peak level and a gene expression levels for the cis-peaks (middle panel, 7,833 pairs) and the trans-peaks (bottom panel, 118.7 million pairs), respectively. X-axes are the SRCCs estimated from the co-assayed cells, which serve as the gold standard, while $Y$-axes are the SRCCs estimated from the pseudo-cells generated by each method. The overall concordance between $X$ and $Y$ are further quantified using a single SRCC shown on the up-left corner of each subfigure. Cis is defined as gene bodies plus 2,000 bps upstream. Reconstructing the gene expression and the TF activity level (Y-axes) of NR3C1 using bindSC pseudo-cells (d). X-axis is the averaged treatment time of the cells in each pseudo-cell. A genome browser view showing putative regulatory relations between an accessible distal site chr2:201770437-201770992 and the gene CFLAR (e). The 6 tracks at the top show ATAC peak levels and gene expression levels at six time points. The track in the middle shows chromatin interactome from published $\mathrm{Hi}-\mathrm{C}$ data. The bottom track shows the NR3C1 binding targets (ChIP-Seq) peaks published in an independent study ${ }^{28}$. 
Fig. 4

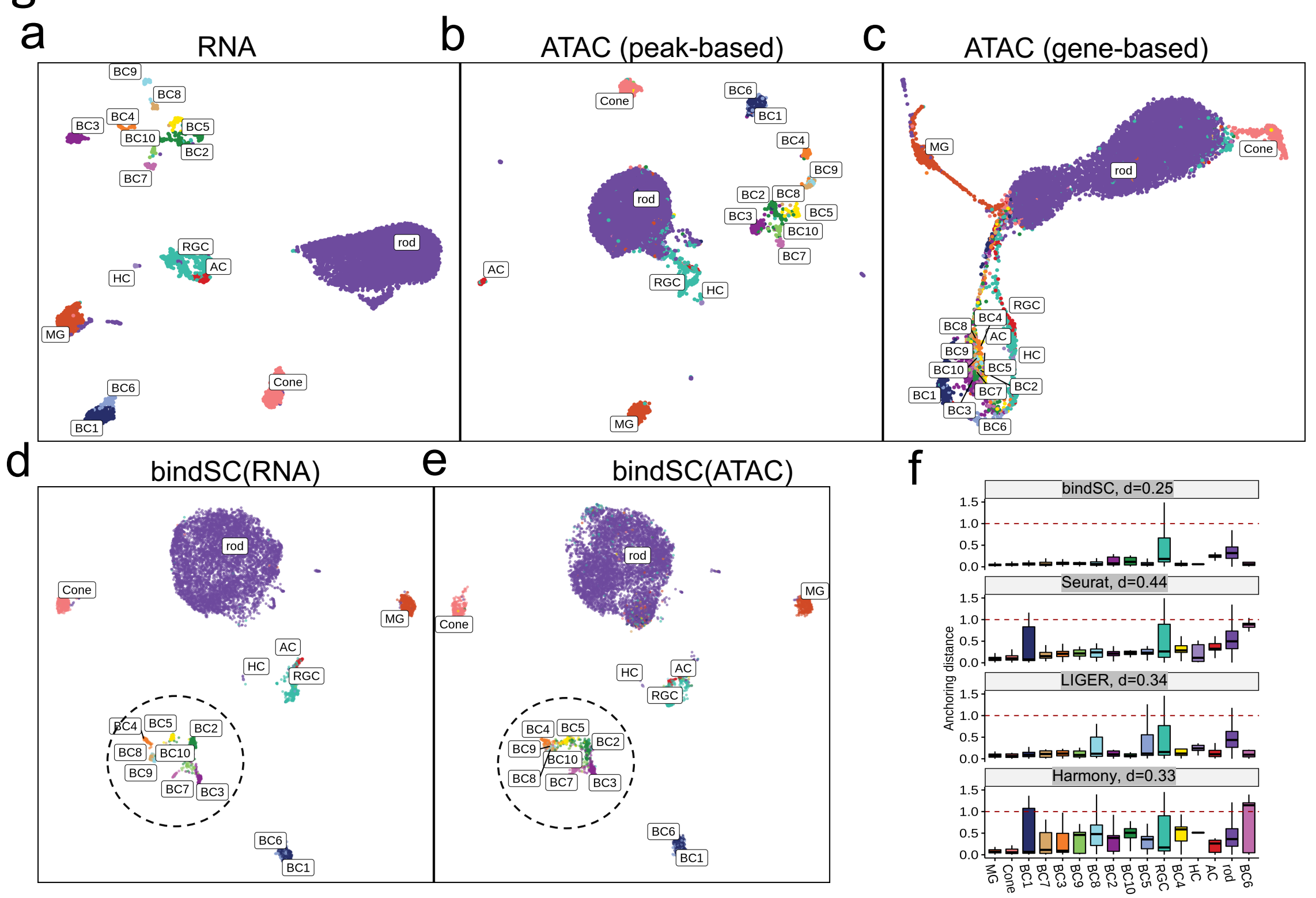

9 bindSC(RNA)

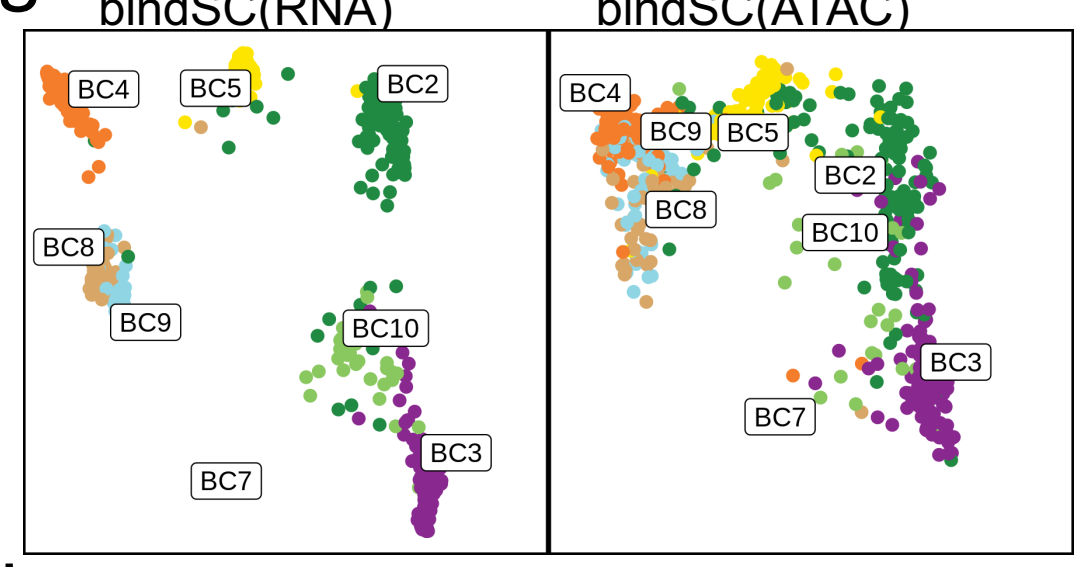

i $\operatorname{LIGER}(\mathrm{RNA})$

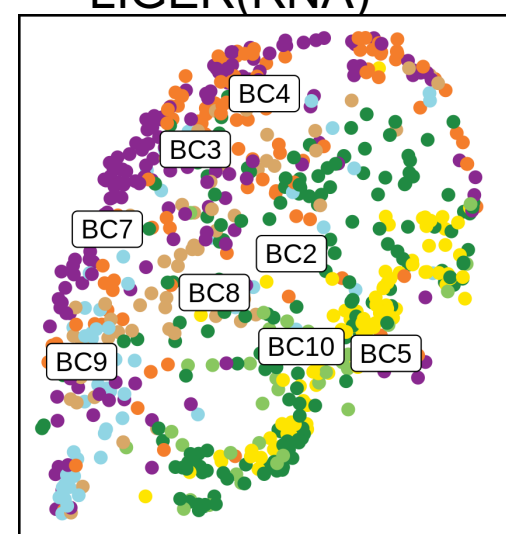

bindSC(ATAC)

LIGER(ATAC)

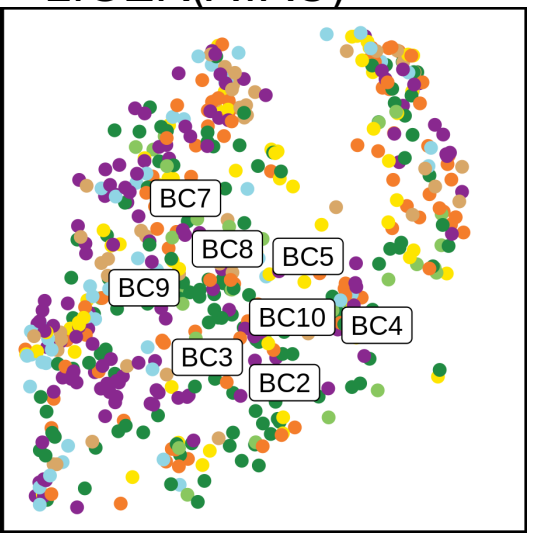

$\mathrm{h}$
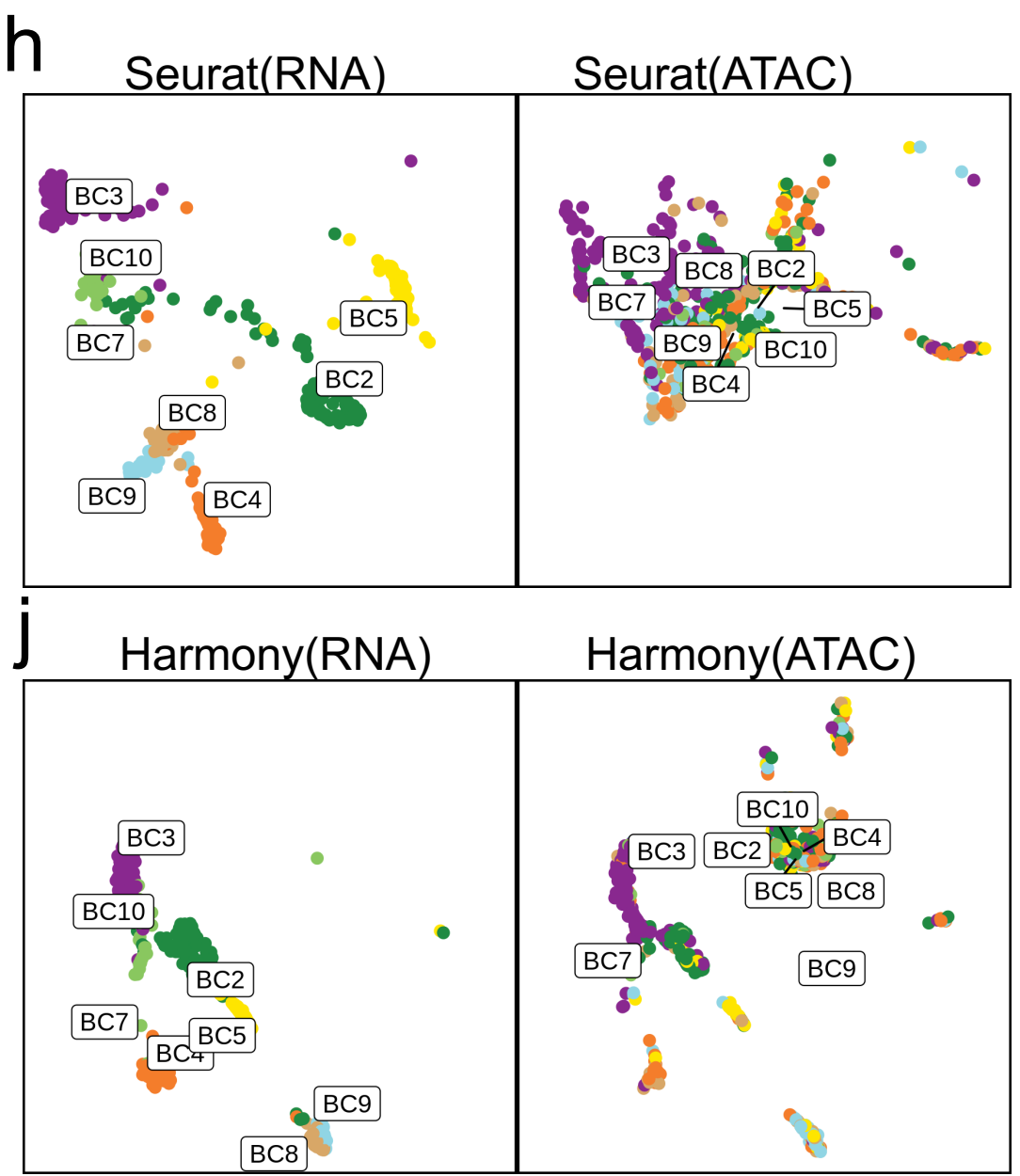

Fig. 4 Integrating single-cell RNA-seq and ATAC-seq on a mouse retinal cell atlas. UMAP views of 9,383 mouse retina cells based on gene expression levels in the RNA-seq data (a), chromatin accessibility peak profiles in the ATAC-seq data (b), gene-level collapsed chromatin accessibility profiles (c). The cells are colored by cell types annotated based on RNA expression levels (Supplementary Fig. 8). BindSC co-embeddings for the cells in the RNA-seq data (d) and those in the ATACseq data (e). Anchoring distances resulting from bindSC, Seurat, LIGER and Harmony (f). The median anchoring distance for each cell type was highlighted as a bold horizonal bar in the middle of each box in each panel. The dotted line denotes the anchoring distance based on random guesses. Zoomed out UMAP views for the BC cells in the co-embeddings generated by bindSC (g), Seurat (h), LIGER (i), and Harmony (j). Integration results for all the cell types can be seen in Supplementary Fig. 9. RGC: retinal ganglion cells; HC: horizontal cells; BC: bipolar cells; AC: amacrine cells; MG: Müller glial cell. 
Fig. 5

a
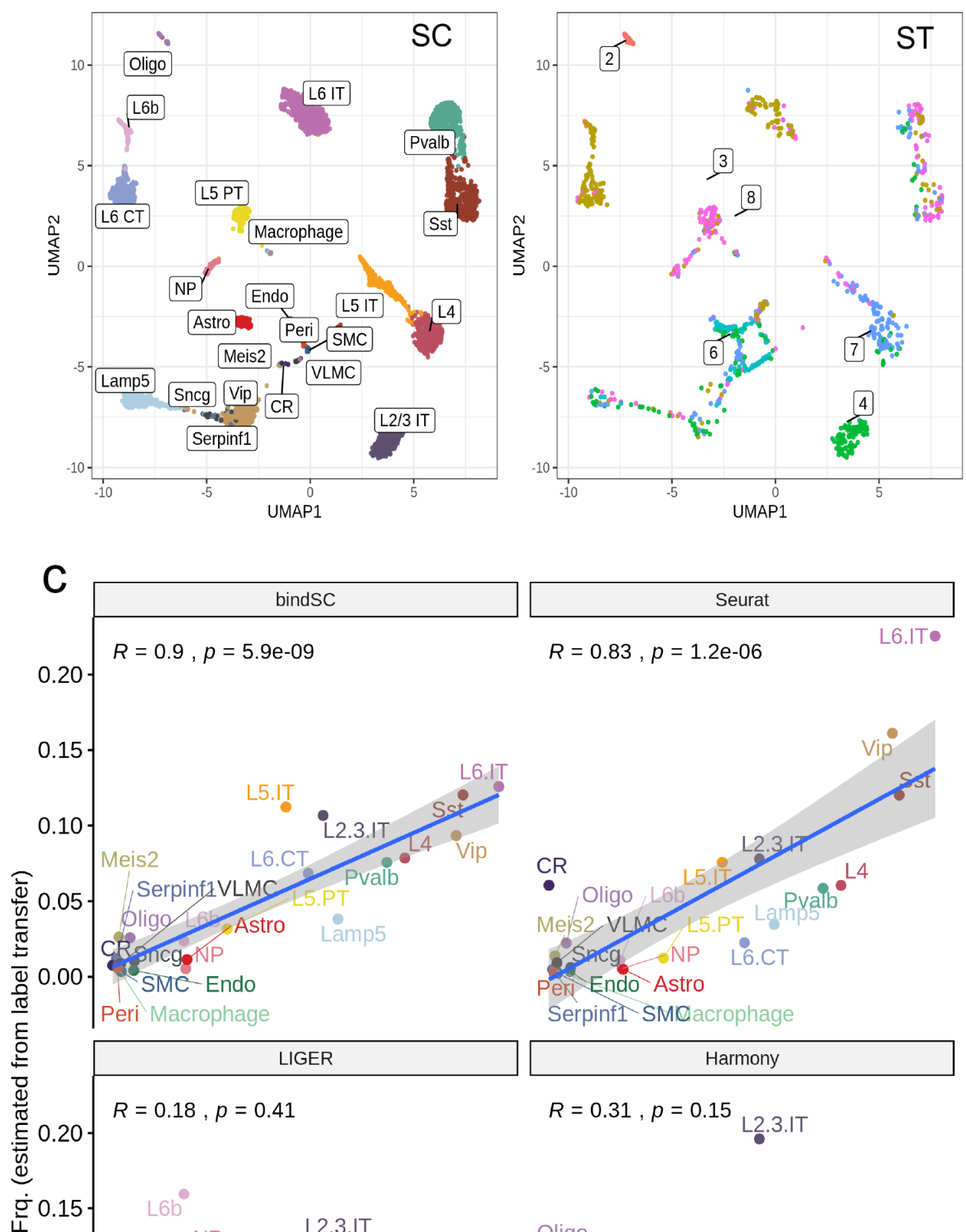

这

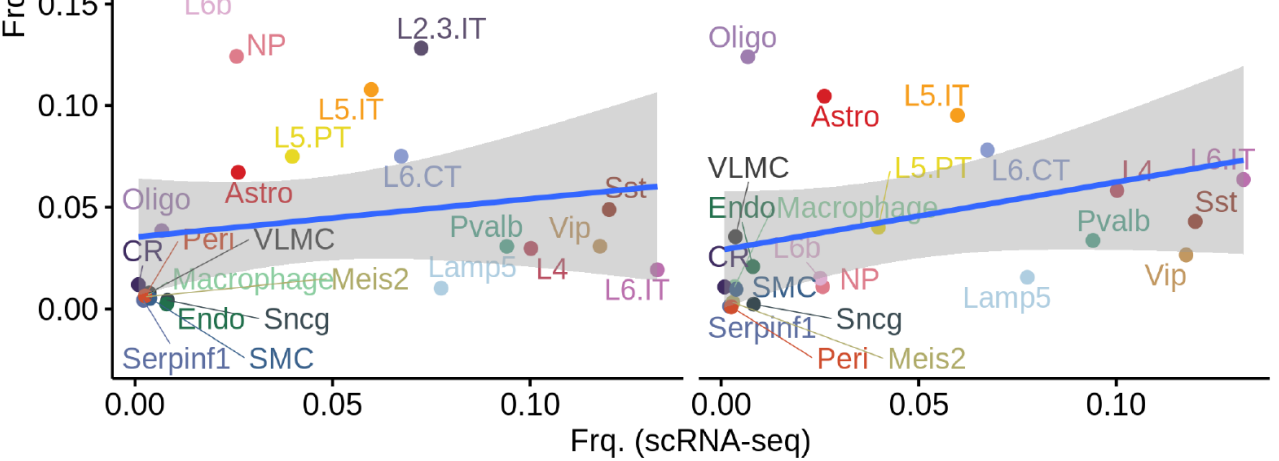

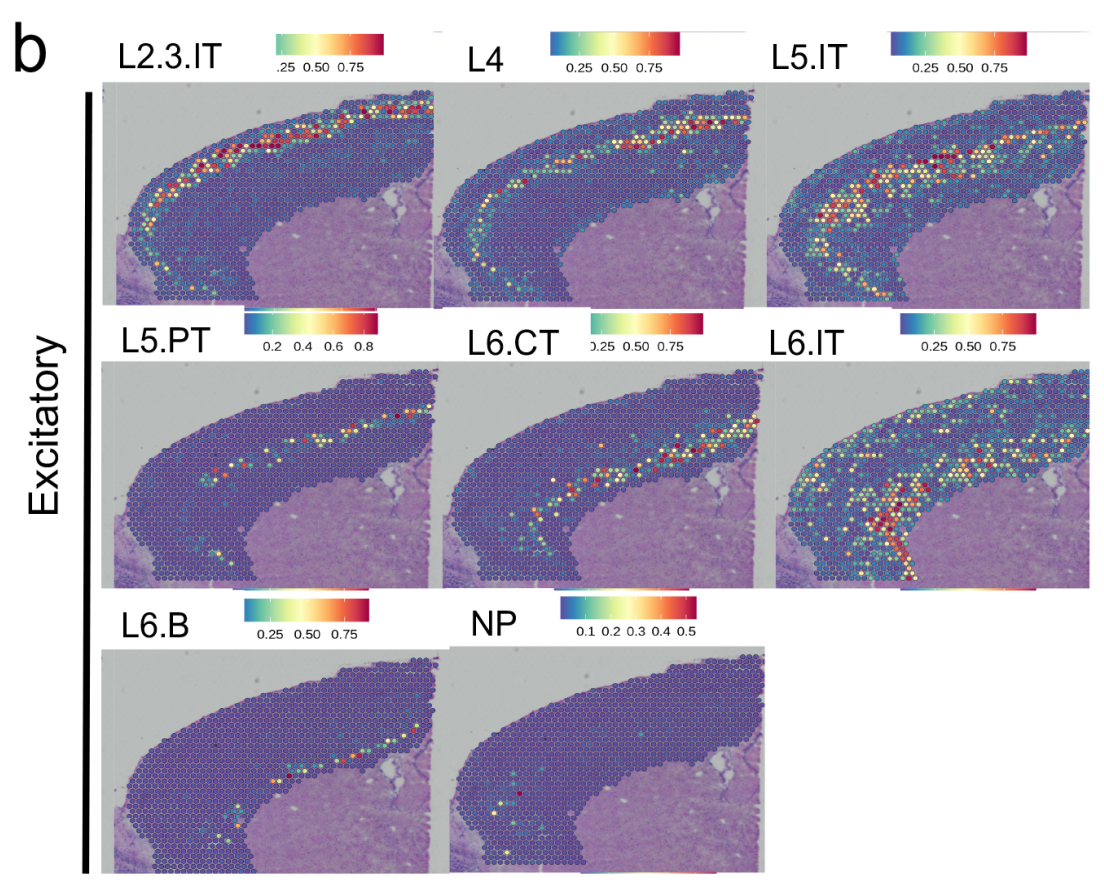
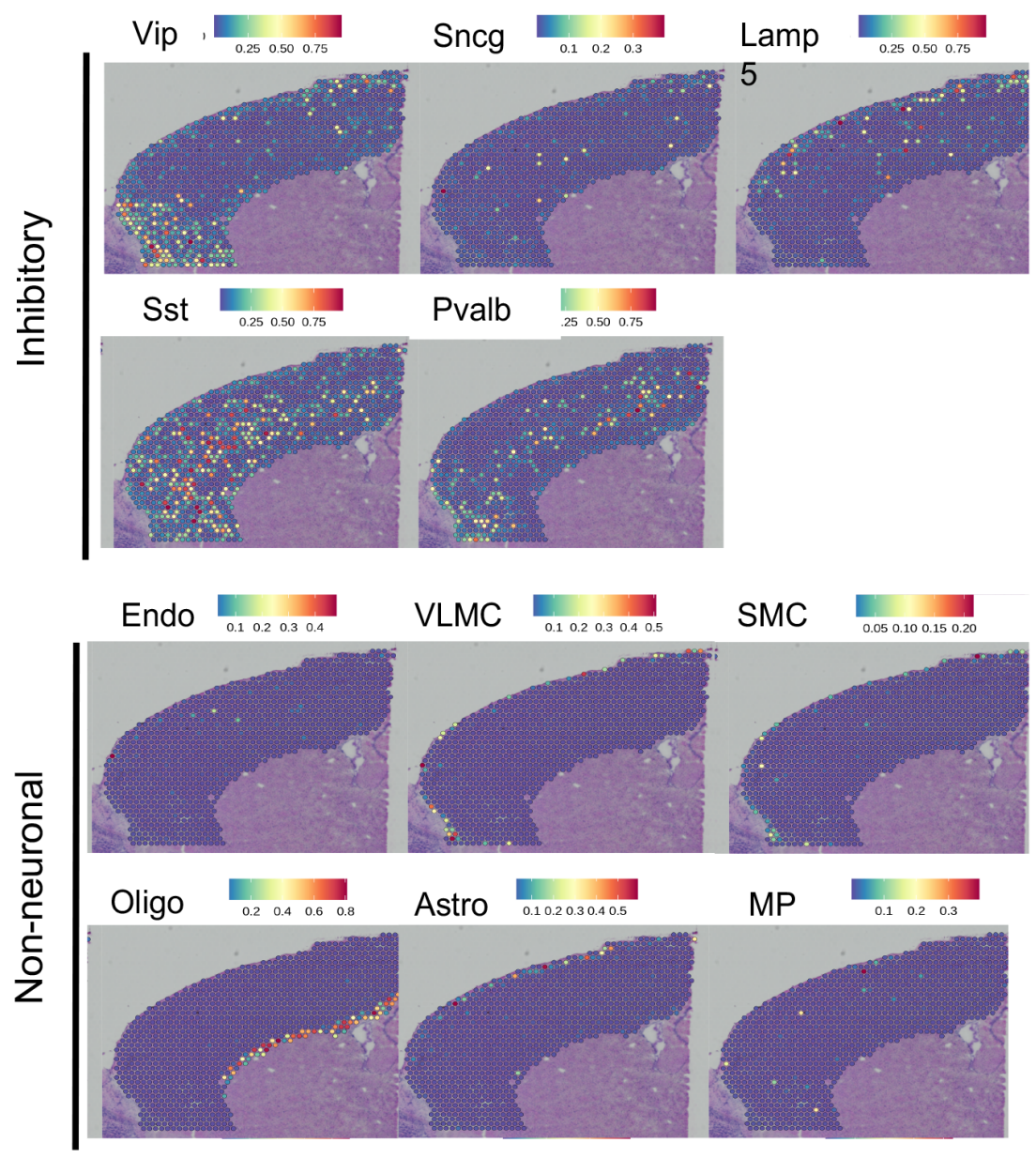

Fig. 5 Integrating spatially resolved transcriptomic (ST) data with scRNA-seq data from mouse frontal cortex. UMAPs of the gene expression levels for the 14,249 cells profiled by SMART-Seq2 and for the 1,072 spots profiled by the 10x Visium technology (a). Cell type labels are from the original publication ${ }^{57}$. Predicted locations of each cell type in the histological images (b). Color gradient corresponds to the probability score of a cell being mapped to a particular spatial location. Comparison of cell type frequencies estimated from the ST data ( $Y$-axis) to those estimated from the scRNA-seq data (X-axis) (c). Correlation coefficients $(R)$ and P values are calculated based on Pearson's correlation analysis. Each dot corresponds to a cell-type (labeled in different colors). The blue line and the grey shade represent regression lines and $95 \%$ confidence intervals from performing linear regressions. 
Fig. 6
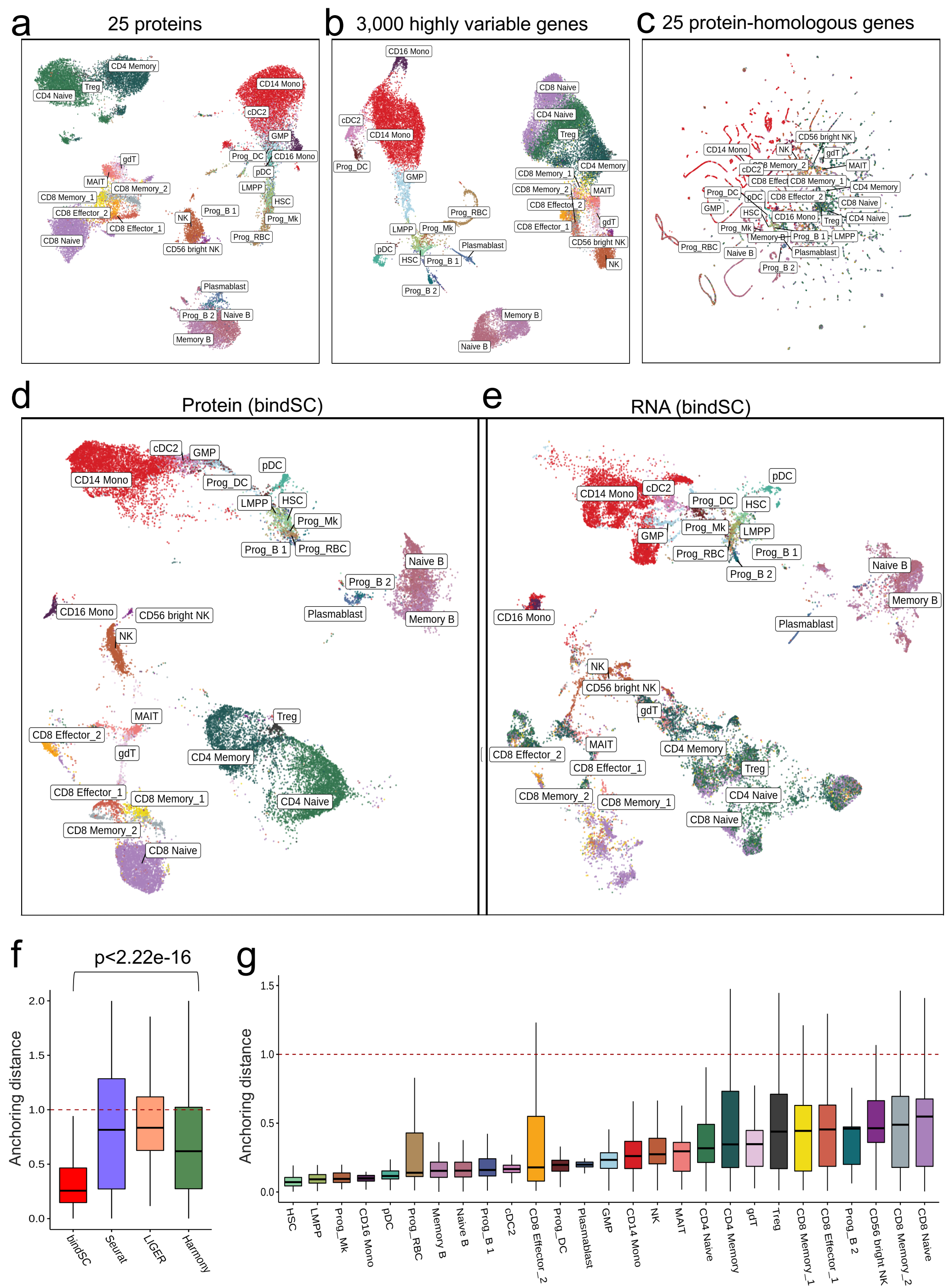

Fig. 6 Integrating single-cell RNA with protein data produced by a CITE-seq assay. The UMAP of 30,672 human bone marrow cells based on 25 surface protein levels (a), 3,000 highly variable gene expression levels (b) and 25 protein-homologous gene expression levels (c). The cell type labels are from the original study ${ }^{17}$. UMAP of the protein (d) and the RNA (e) expression data in the co-embedding generated by bindSC. Comparison of anchoring distances generated by bindSC, Seurat, LIGER and Harmony (f). The red dotted line denotes the anchoring distance from random guesses. Anchoring distances for each cell type in the bindSC co-embedding (g). 